\title{
Article
}

\section{On solar system dynamics in general relativity}

Battista, Emmanuele, Esposito, Giampiero, Di Fiore, Luciano, Dell'Agnello, Simone, Simo, Jules and Grado, Aniello

Available at http://clok.uclan.ac.uk/18653/

Battista, Emmanuele, Esposito, Giampiero, Di Fiore, Luciano, Dell'Agnello, Simone, Simo, Jules ORCID: 0000-0002-1489-5920 and Grado, Aniello (2017) On solar system dynamics in general relativity. International Journal of Geometric Methods in Modern Physics (ijgmmp), 14 (9). pp. 1750117-1. ISSN 0219-8878

It is advisable to refer to the publisher's version if you intend to cite from the work. DOI: 10.1142/S0219887817501171

For more information about UCLan's research in this area go to http://www.uclan.ac.uk/researchgroups/ and search for <name of research Group>.

For information about Research generally at UCLan please go to http://www.uclan.ac.uk/research/

All outputs in CLoK are protected by Intellectual Property Rights law, including Copyright law. Copyright, IPR and Moral Rights for the works on this site are retained by the individual authors and/or other copyright owners. Terms and conditions for use of this material are defined in the policies page.

\section{CLoK}

Central Lancashire online Knowledge www.clok.uclan.ac.uk

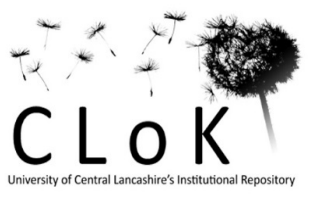




\title{
On solar system dynamics in general relativity
}

\author{
Emmanuele Battista, ${ }^{*}$ Giampiero Esposito ${ }^{\dagger}$ and Luciano Di Fiore ${ }^{\ddagger}$ \\ Istituto Nazionale di Fisica Nucleare, Sezione di Napoli, \\ Complesso Universitario di Monte S. Angelo, Via Cintia Edificio 6, 80126 Napoli, Italy \\ Simone Dell'Agnello $§$ \\ Istituto Nazionale di Fisica Nucleare, Laboratori Nazionali di Frascati, 00044 Frascati, Italy \\ Jules Simo** \\ Aerospace Engineering, Computing 85 Technology Building, \\ School of Engineering, University of Central Lancashire, Preston, PR1 2HE, United Kingdom
}

\author{
Aniello Grado ${ }^{\dagger \dagger}$ \\ INAF, Osservatorio Astronomico di Capodimonte, 80131 Napoli, Italy
}

(Dated: October 4, 2016)

\begin{abstract}
Recent work in the literature has advocated using the Earth-Moon-planetoid Lagrangian points as observables, in order to test general relativity and effective field theories of gravity in the solar system. However, since the three-body problem of classical celestial mechanics is just an approximation of a much more complicated setting, where all celestial bodies in the solar system are subject to their mutual gravitational interactions, while solar radiation pressure and other sources of nongravitational perturbations also affect the dynamics, it is conceptually desirable to improve the current understanding of solar system dynamics in general relativity, as a first step towards a more accurate theoretical study of orbital motion in the weak-gravity regime. For this purpose, starting from the Einstein equations in the de Donder-Lanczos gauge, this paper arrives first at the Levi-Civita Lagrangian for the geodesic motion of planets, showing in detail under which conditions the effects of internal structure and finite extension get cancelled in general relativity to first post-Newtonian order. The resulting nonlinear ordinary differential equations for the motion of planets and satellites are solved for the Earth's orbit about the Sun, written down in detail for the Sun-Earth-Moon system, and investigated for the case of planar motion of a body immersed in the gravitational field produced by the other bodies (e.g. planets with their satellites). At this stage, we prove an exact property, according to which the fourth-order time derivative of the original system leads to a linear system of ordinary differential equations. This opens an interesting perspective on forthcoming research on planetary motions in general relativity within the solar system, although the resulting equations remain a challenge for numerical and qualitative studies. Last, the evaluation of quantum corrections to location of collinear and noncollinear Lagrangian points for the planar restricted three-body problem is revisited, and a new set of theoretical values of such corrections for the Earth-Moon-planetoid system is displayed and discussed. On the side of classical values, the general relativity corrections to Newtonian values for collinear and noncollinear Lagrangian points of the Sun-Earth-planetoid system are also obtained. A direction for future research will be the analysis of planetary motions within the relativistic celestial mechanics set up by Blanchet, Damour, Soffel and $\mathrm{Xu}$.
\end{abstract}

PACS numbers: 04.60.Ds, 95.10.Ce

\section{INTRODUCTION}

Recent work by the authors [1-4], motivated, on the quantum side, by modern developments in effective field theories of gravity [5-13], and, on the classical side, by the beautiful discoveries in celestial mechanics [14-69], aerospace engineering [70-77] and (lunar) laser ranging techniques [78-90], has studied in detail the libration points of the

\footnotetext{
*E-mail: ebattista@na.infn.it

${ }^{\dagger}$ E-mail: gesposit@na.infn.it

${ }^{\ddagger}$ E-mail: luciano.difiore@na.infn.it

$\S$ E-mail: simone.dellagnello@lnf .infn.it

**E-mail: JSimo@uclan.ac.uk

††E-mail: agrado@na.astro.it
} 
restricted three-body problem in Newtonian gravity, general relativity and effective field theories of gravity. In particular, we have found (see Ref. [4] and Erratum therein) that general relativity corrects by $0.19 \mathrm{~mm},-0.32 \mathrm{~mm}$ and $-0.04 \mathrm{~mm}$ the location of the collinear Lagrangian points $L_{1}, L_{2}$ and $L_{3}$ respectively, for the circular restricted three-body problem where a planetoid (e.g. a satellite) moves in the gravitational field of the Earth and the Moon. Moreover, for the planar $(x, y)$ coordinates of noncollinear Lagrangian points $L_{4}$ and $L_{5}$, the Einstein correction to Newtonian values is $2.73 \mathrm{~mm}$ for $x$ and $-1.59 \mathrm{~mm}$ for $y$. The possible quantum corrections ${ }^{1}$ to the Lagrangian points $L_{1} \ldots L_{5}$ are just below or just above $1 \mathrm{~mm}$. These tiny theoretical values are conceptually interesting but, unfortunately, too small to put on firm ground the hope that one might arrive in the near future to a new test of general relativity or to a clearcut discrimination between corrections predicted by general relativity and those predicted by effective field theories of gravity.

Nevertheless, since the three-body problem is just an approximation of a much more complicated setting, where all celestial bodies in the solar system (planets, satellites, asteroids, ...) interact with each other while solar radiation pressure [91] and other sources of nongravitational perturbations may also come into play, it remains conceptually desirable to improve the current understanding of solar system dynamics in general relativity, as a first step towards a more accurate theoretical study of orbital motion, possibly including the outstanding open problem of solar system stability.

For this purpose, Sec. II performs a concise but detailed review of the $N$-body problem in general relativity, following the thorough analysis in Ref. [20]. First, the Einstein equations are studied in the de Donder-Lanczos gauge so as to obtain a Lagrangian for the geodesic motion of celestial bodies. Second, the physics of gravitating bodies with finite extension is studied, showing in detail under which conditions one arrives at a cancellation effect also in classical general relativity. Section III applies the Lagrangian of Sec. II to the Earth's motion around the Sun and then to the Sun-Earth-Moon system, and eventually to the planar motion of a body subject to the gravitational attraction of several bodies, when all their mutual distances are large enough that we are in the weak-gravity regime. Section IV derives in detail the linear (not linearized!) system of ordinary differential equations associated with our nonlinear equations of motion. Section V considers another possible definition of quantum corrections to Lagrangian points. Results and open problems are discussed in Sec. VI.

\section{LAGRANGIAN OF THE N-BODY PROBLEM IN GENERAL RELATIVITY}

In Ref. [20], the N-body Lagrangian of celestial mechanics in general relativity is derived by appealing to the geodesic principle for the motion of each celestial body. However, a crucial clarification should be made before we go on. The two-body analysis in Ref. [20] is completely superseded, for a number of technical reasons well described in Refs. [28, 31, 61], mainly related to an over-simplified analysis of the internal structure of the bodies. If one wants to study a generic $N$-body system, the technique in Refs. [33-36] is both conceptually and technically superior. It relies on a global chart for describing the overall dynamics of the $N$ bodies, and $N$ local charts adapted to the separate description of the structure and environment of each body. Within that framework, the gravitational field locally generated by each body is expressed by the Blanchet-Damour [27] relativistic multipole moments, while the external gravitational field experienced by each body is expanded in terms of a set of relativistic tidal moments. Even the geodesic motion is proved, rather than being assumed, for a system of mass monopoles. However, the Levi-Civita analysis of the $N$-body problem remains of some value. Indeed, in Ref. [28] the author writes: "... It was remarked by M. Brillouin that the arguments by which one can efface the internal structure in Newton's theory cannot be immediately translated into Einstein's theory. This problem has been clearly formulated by Levi-Civita. He proved by detailed arguments within Einstein's theory that this effacement property was still valid at the first post-Newtonian approximation, in the sense that all large direct self-action effects cancel or contribute terms in the equations of motion which can be renormalized away, so that the final equations of motion can be written in terms of only some centers of mass and some effective masses." Moreover, in Ref. [31] the authors write: "... In conclusion, we may say that Levi-Civita was the first to stress the need for setting up a mathematically consistent framework for the general relativistic problem of $n$ weakly self-gravitating bodies, including a clear treatment of self-field effects. ... Concerning the general framework, in his book, he gave a careful presentation of the many subtle (mathematical and physical) issues involved in the relativistic problem of motion. This beautiful model of what a relativistic theory of the $n$-body problem should look like has, alas, rarely been matched by subsequent works in this field. Particularly remarkable is Levi-Civita's clear recognition of the importance of the effacement properties of self-field effects in general relativity."

\footnotetext{
1 These might be seen as low-energy effects in quantum gravity, more easily accessible than the elusive high-energy effects of the Planck era.
} 
As will be clear from the synthesis we provide below, the Levi-Civita assumptions remain useful for studying not a generic system of $N$ arbitrarily composed and shaped, weakly self-gravitating, rotating, deformable bodies, nor the binary systems of relativistic astrophysics, but precisely the planetary motions in the solar system. When tested in the well-known case of the Earth's motion about the Sun, they pass this first consistency check, but the resulting $N$-planet Lagrangian is extremely complicated, despite the simplifying assumptions. The technique in Refs. [33-36] has led, over three decades, to unprecedented and unmatched calculational power for two-body systems and the associated effective-one-body formalism $[42,44,50,52,54,55,64-67]$, but the resulting equations of motion for all planets with their satellites, including the relativistic multipole and tidal moments, are a challenging task, even for modern computers. For example, in Ref. [36], to discuss in more detail the post-Newtonian acceleration of an Earth-orbiting satellite, the authors make several approximations in the formula for the total acceleration. Thus, the scheme that we are going to present can be seen as an intermediate step between the Newtonian mechanics of the solar system and its complete theory in general relativity.

The first part of the analysis reported hereafter deals with the field equations and derives an approximate form of the metric components and of the resulting line element under suitable assumptions. The second part of the analysis relies upon three basic assumptions on the physics of gravitating systems, which make it precise what sort of cancellation principle still holds on passing from Newtonian to relativistic celestial mechanics. Hence a Lagrangian of $N$ material points is eventually derived.

\section{A. The energy-momentum tensor}

In Ref. [20], Levi-Civita considered eventually a granular (or perfect) matter with energy-momentum tensor

$$
T_{\mu \nu}=\varepsilon \lambda_{\mu} \lambda_{\nu}
$$

where $\lambda_{\mu}$ is defined by $\lambda_{\mu} \equiv \frac{\mathrm{d} x_{\mu}}{\mathrm{d} s}, s$ being the affine parameter describing the congruence of world lines (or geodesics, see below). He arrived at this by starting from a more general form of the tensor $T$, defined by the formula

$$
T_{\mu \nu}=(\varepsilon+p) \lambda_{\mu} \lambda_{\nu}-p g_{\mu \nu},
$$

where $p$ is an isotropic pressure. In other words, to ensure the individual character and consistency of celestial bodies, internal stresses play a key role, and one considers a scheme where the internal stresses are introduced in the particularly simple form of an isotropic pressure. The congruence of world lines is eventually geodesic, and this property depends on the canonical form $\varepsilon \lambda_{\mu} \lambda_{\nu}$ of the tensor $T$ [20]. The logical steps leading to Eq. (2.1) are as follows [20].

By virtue of Eq. (2.2), the contribution of pressure to the trace of the energy-momentum tensor is

$$
T^{*}=p \sum_{\mu, \nu=0}^{3} g^{\mu \nu}\left(\lambda_{\mu} \lambda_{\nu}-g_{\mu \nu}\right)=-3 p
$$

and the contribution of pressure to the tensor $\tau_{\mu \nu} \equiv T_{\mu \nu}-T g_{\mu \nu}$ is

$$
\tau_{\mu \nu}^{*}=p\left(\lambda_{\mu} \lambda_{\nu}-g_{\mu \nu}\right)-T^{*} g_{\mu \nu}=p\left(\lambda_{\mu} \lambda_{\nu}+2 g_{\mu \nu}\right) .
$$

It is useless to take into account these additional terms in the differential equations (with the notation of Ref. [20], $\triangle_{2}^{0}$ is the Laplacian evaluated according to an Euclidean metric, $\gamma_{\mu \nu}$ are metric perturbations and $\kappa \equiv \frac{8 \pi G_{N}}{c^{4}}$ )

$$
\triangle_{2}^{0} \gamma_{\mu \nu}=-\kappa \tau_{\mu \nu}
$$

when $\mu, \nu$ are not simultaneously 0 because one can neglect the terms higher than the first order and multiplied by $\varepsilon$. That results immediately from the fact that the ratio

$$
\frac{p}{\varepsilon}=\frac{1}{c^{2}} \frac{p}{\mu}
$$

can be seen as a quantity of first order, where

$$
\frac{p}{\mu}=\left(v_{s}\right)^{2}<\left(v_{\text {celestial bodies }}\right)^{2} .
$$


On the contrary, it is important to take into account the additional terms in the equation

$$
\triangle_{2}^{0} \gamma_{00}=-\kappa \tau_{00}
$$

because we need $\gamma_{00}$ up to second order. The pressure contribution to $\tau_{00}$ is

$$
\tau_{00}^{*}=p\left(\left(\lambda_{0}\right)^{2}+2 g_{00}\right)
$$

Thus, the additional term on the right-hand side of Eq. (2.8) can be written as

$$
-\kappa p\left(\left(\lambda_{0}\right)^{2}+2 g_{00}\right)=-3 \kappa \varepsilon \frac{p}{\varepsilon}(1+\text { second }- \text { order terms }) .
$$

One then finds a unique potential for each of the bodies having density

$$
\varepsilon^{\prime} \equiv \varepsilon\left(1-3 \frac{p}{\varepsilon}\right)
$$

Hence the Poisson equations defining the 10 potentials $\gamma_{\mu \nu}$ are exactly the same we had in absence of pressure, provided one replaces $\varepsilon$ by $\varepsilon^{\prime}$ according to Eq. (2.11).

As a next step, since the left-hand side of the Einstein equations is purely geometric and obeys the Bianchi identities, its coupling to the energy-momentum tensor of matter enforces 4 conservation equations, and one has to understand how the pressure modifies such equations. Indeed, by virtue of Eq. (2.2), one finds

$$
\sum_{\nu=0}^{3} \nabla^{\nu} T_{\nu \mu}=0 \Longrightarrow(\varepsilon+p) k_{\mu}-\lambda_{\mu}\left[\frac{\mathrm{d}}{\mathrm{d} s}(\varepsilon+p)+(\varepsilon+p) \operatorname{div} \lambda\right]-p_{\mu}=0
$$

where $k_{\mu} \equiv \sum_{\nu=0}^{3} \lambda^{\nu} \nabla_{\mu} \lambda_{\nu}$ are covariant components of geodesic curvature of worldlines. One can exploit the property of $k_{\mu}$ of being orthogonal to the worldlines, i.e.

$$
\sum_{\mu=0}^{3} k_{\mu} \lambda^{\mu}=0
$$

upon multiplying Eq. (2.12) by $-\lambda^{\mu}$ and then summing over all values of $\mu$, hence finding

$$
\frac{\mathrm{d} \varepsilon}{\mathrm{d} s}+\frac{\mathrm{d} p}{\mathrm{~d} s}+(\varepsilon+p) \operatorname{div} \lambda+\sum_{\mu=0}^{3} p_{\mu} \lambda^{\mu}=0
$$

In this equation, $\frac{\mathrm{d} p}{\mathrm{~d} s}$ vanishes because the pressure is taken to be constant along a worldline (this assumption is satisfied by planets, which behave as solids), and $\sum_{\mu=0}^{3} p_{\mu} \lambda^{\mu}$ vanishes as well by assumption on the pressure. Thus, Eq. (2.14) reduces to

$$
(\varepsilon+p) k_{\mu}-p_{\mu}=0
$$

In this equation, the 0 component is identically satisfied, but in the spatial components the local influence of pressure is not in general negligible, because one finds

$$
k_{i}=\frac{p_{i}}{(\varepsilon+p)} \forall i=1,2,3 \Longrightarrow k=(\varepsilon+p)^{-1} \operatorname{grad} p .
$$

However, if one considers the center of gravity (see Sec. II.D) of a body, provided the symmetry condition is still satisfied, the gradient of pressure vanishes for the center of gravity. With this understanding, we can set $k_{i}=0 \forall i=$ $1,2,3$, which implies that the geodesic principle still holds, and it is therefore legitimate to limit ourselves, in a first approximation, to unbundled media [20].

These calculations show that our source [20] considered perfect matter only after a number of detailed calculations supplemented by physical assumptions, but of course are not a proof that one has to follow this conceptual path. In the following subsections we are going to get a detailed understanding of the resulting Lagrangian for planetary motion. 


\section{B. The Einstein equations}

The Einstein equations

$$
R_{\mu \nu}-\frac{1}{2} g_{\mu \nu} R=\frac{8 \pi G_{N}}{c^{4}} T_{\mu \nu}
$$

are a quasi-linear set of partial differential equations. As is well known, this means that they are linear in second-order derivatives of the metric $g_{\mu \nu}$, whereas the nonlinearity results from the squares of first derivatives of the spacetime metric. Such a quasi-linear system is not in normal form unless a suitable supplementary condition (more frequently called gauge-fixing) is imposed. In the Levi-Civita analysis we rely upon, such a condition is the so-called de DonderLanczos gauge. The Lanczos gauge sets to zero the action of the scalar wave operator $\square$ on spacetime coordinates, i.e.

$$
\square x^{\nu}=-\sum_{\lambda, \mu=0}^{3} g^{\lambda \mu} \Gamma_{\lambda \mu}^{\nu}=0 .
$$

Such a set of spacetime coordinates, if they exist, were said to be isometric because Eqs. (2.2) generalize the harmonic character of Cartesian coordinates in a Euclidean metric. The de Donder formulation takes linear combinations of such equations with coefficients given by covariant components of the metric, i.e.

$$
Z_{\mu}=\sum_{\nu=0}^{3} g_{\mu \nu} \square x^{\nu}
$$

Now a remarkable identity relates the Ricci tensor and the symmetrized partial derivatives of the $Z_{\mu}$, i.e.

$$
R_{\mu \nu}-\frac{1}{2}\left(\frac{\partial Z_{\mu}}{\partial x^{\nu}}+\frac{\partial Z_{\nu}}{\partial x^{\mu}}\right)=\frac{1}{2} \sum_{\lambda, \rho=0}^{3} g^{\lambda \rho} \frac{\partial^{2} g_{\mu \nu}}{\partial x^{\lambda} \partial x^{\rho}}+F\left(g_{\alpha \beta}, \frac{\partial g_{\mu \nu}}{\partial x^{\rho}}\right)
$$

The term $F$ is of lower order in that it depends only on the metric and its first partial derivatives. In the desired isometric coordinates, for which the $Z_{\mu}$ vanish, the Einstein equations, jointly with the conservation equations for the energy-momentum tensor resulting from the Bianchi identity, take therefore the normal form [20] with respect to the $x^{0}$ variable.

In the following analysis, the dimensionless terms with an order of magnitude of

$$
\beta^{2} \equiv \frac{v^{2}}{c^{2}}, \gamma \equiv \frac{G_{N}}{c^{2}} \int_{S} \frac{\mu}{r} \mathrm{~d} S=\frac{G_{N}}{c^{2}} \sum_{h=1}^{n} \int_{h} \frac{\mu}{r} \mathrm{~d} C_{h}
$$

where $S$ is the region occupied by all bodies $C_{h}, h=1, \ldots, n$, and $\mu$ is the function representing ${ }^{2}$ the local density, are said to be of first order and are denoted by $\mathrm{O}(I)$. The use of the de Donder-Lanczos gauge implies that the metric components take the approximate form [20]

$$
\begin{gathered}
g_{00}=1-2 \gamma+2 \gamma^{2}-2 \zeta, \\
g_{0 i}=g_{i 0}=4 \gamma_{i}+\mathrm{O}(I I), \forall i=1,2,3, \\
g_{i i}=-1-2 \gamma, \forall i=1,2,3 .
\end{gathered}
$$

With this notation, $\gamma$ is the Newtonian potential in a domain $S$ of Euclidean space ${ }^{3}$ with Cartesian coordinates $x^{1}, x^{2}, x^{3}$, and $\mathrm{O}(I I)$ is a term of second order in $\beta^{2}$ and $\gamma$. If $x^{0} \equiv c t$, the associated dimensionless velocities are

$$
\beta^{i} \equiv \frac{\mathrm{d} x^{i}}{\mathrm{~d} x^{0}} \Longrightarrow \beta^{2} \equiv \sum_{i=1}^{3}\left(\beta^{i}\right)^{2}
$$

\footnotetext{
2 No confusion should arise when we write instead $\mu$ for a coordinate index in tensor equations, and similarly for other Greek letters used hereafter, whose meaning will be clear from the context.

3 It is a nontrivial property that three-dimensional space is taken to be Euclidean. From the point of view of general formalism, some authors regard this assumption as Procuste's bed [28] and hence a severe drawback, but for the analysis of planetary motions this remains a legitimate approach, whereas it would be totally inappropriate in the relativistic astrophysics of binary systems.
} 
One can therefore define the three potentials

$$
\gamma_{i} \equiv \frac{G_{N}}{c^{2}} \int_{S} \frac{\mu \beta_{i}}{r} \mathrm{~d} S
$$

while the function $\zeta$ can be split into the sum of three functions, i.e. [20]

$$
\zeta \equiv \varphi+\psi+\nu
$$

where $\varphi$ and $\psi$ solve a Poisson-type equation:

$$
\begin{aligned}
\varphi & \equiv-\frac{G_{N}}{c^{2}} \int_{S} \frac{\mu \gamma}{r} \mathrm{~d} S, \\
\psi & \equiv \frac{3}{2} \frac{G_{N}}{c^{2}} \int_{S} \frac{\mu \beta^{2}}{r} \mathrm{~d} S,
\end{aligned}
$$

whereas

$$
\nu \equiv \frac{1}{2} \frac{G_{N}}{c^{2}} \frac{\partial^{2}}{\partial\left(x^{0}\right)^{2}} \int_{S} \mu r \mathrm{~d} S
$$

By virtue of (2.22)-(2.30), the squared line element takes the approximate form

$$
\left(\frac{\mathrm{d} s}{\mathrm{~d} x^{0}}\right)^{2}=1-2\left(\frac{\beta^{2}}{2}+\gamma\right)+2 \gamma^{2}-2 \zeta-2 \gamma \beta^{2}+8 \sum_{i=1}^{3} \gamma_{i} \beta^{i} .
$$

The formulation of the geodesic principle for celestial bodies needs actually the square root of (2.31), i.e.

$$
\delta \int \mathrm{d} s=\delta \int \frac{\mathrm{d} s}{\mathrm{~d} x^{0}} \mathrm{~d} x^{0}=0
$$

From the standard second-order Taylor expansion of $(1+\varepsilon)^{\frac{1}{2}}$ in the neighboorhood of $\varepsilon=0$ one gets, upon denoting by $\mathcal{N}$ the Newtonian gravitational Lagrangian

$$
\mathcal{N} \equiv \frac{\beta^{2}}{2}+\gamma
$$

the useful approximate formula

$$
\begin{aligned}
\frac{\mathrm{d} s}{\mathrm{~d} x^{0}} & =\sqrt{1-2\left(\frac{\beta^{2}}{2}+\gamma\right)+2 \gamma^{2}-2 \zeta-2 \gamma \beta^{2}+8 \sum_{i=1}^{3} \gamma_{i} \beta^{i}} \\
& \approx 1-\mathcal{N}+\gamma^{2}-\zeta-\gamma \beta^{2}+4 \sum_{i=1}^{3} \gamma_{i} \beta^{i}-\frac{1}{2} \mathcal{N}^{2} .
\end{aligned}
$$

The approximate calculation leading from (2.31) to (2.34) is simple, but it represents a crucial conceptual step. A rigorous analysis of stability of the solar system would require working with the square root on the first line of Eq. (2.34) without any expansion, and then using the modern qualitative methods of the calculus of variations. The constant 1 is of course inessential, and this is made precise be pointing out that also the following variation vanishes [92]:

$$
\delta \int \mathrm{d} x^{0}=0,
$$

because we can avoid letting $x^{0}$ to vary since the left-hand side of the equation expressing the geodesic principle undergoes a variation that vanishes by virtue of the conditions resulting from variation of the three coordinates $x^{1}, x^{2}, x^{3}$. In light of (2.32) and (2.35), we get eventually the dimensionless Lagrangian

$$
\mathcal{L}=1-\frac{\mathrm{d} s}{\mathrm{~d} x^{0}}=\mathcal{N}+\mathcal{D}
$$

where $\mathcal{N}$, defined in (2.33), is the Lagrangian of a dimensionless material element in Newtonian mechanics, whereas $\mathcal{D}$ is the Einstein modification of this Newtonian Lagrangian, and is given by [20]

$$
\mathcal{D} \equiv \frac{1}{2} \mathcal{N}^{2}-\gamma^{2}+\zeta+\gamma \beta^{2}-4 \sum_{i=1}^{3} \gamma_{i} \beta^{i}
$$




\section{Physics of gravitating bodies with finite extension}

The Einstein modification $\mathcal{D}$ in $(2.37)$ contains the functions $\gamma, \gamma_{i}, \zeta$ obtained by integrating over all bodies, and hence is related to finite size and internal structure of such bodies, that we will later identify with Sun, Earth, Moon, all planets with their satellites, and a mechanical satellite sent off from the Earth. Following Ref. [20], for each body we can think of the domain $S$ as the disjoint union of $C$ with $S^{\prime}$, where $C$ denotes the domain occupied by the body, while $S^{\prime}$ is the residual portion of $S$. We shall use hereafter the notation according to which

$$
\begin{gathered}
\left.\gamma^{\prime} \equiv \gamma\right|_{S^{\prime}},\left.\gamma^{\prime \prime} \equiv \gamma\right|_{C}, \\
\left.\zeta^{\prime} \equiv \zeta\right|_{S^{\prime}},\left.\zeta^{\prime \prime} \equiv \zeta\right|_{C},
\end{gathered}
$$

so that we can write the decompositions

$$
\gamma=\gamma^{\prime}+\gamma^{\prime \prime}, \zeta=\zeta^{\prime}+\zeta^{\prime \prime}
$$

and also, for the two parts (Newton and Einstein) of the full Lagrangian, the resulting splits

$$
\mathcal{N}=\mathcal{N}^{\prime}+\mathcal{N}^{\prime \prime}, \mathcal{D}=\mathcal{D}^{\prime}+\mathcal{D}^{\prime \prime},
$$

where $\mathcal{N}^{\prime}, \mathcal{D}^{\prime}$ are the part that we would have if the body $C$ were suppressed, whereas $\mathcal{N}^{\prime \prime}, \mathcal{D}^{\prime \prime}$ characterize the influence of the body $C$ on the motion of a point $P \in C$. The explicit formulas we need are

$$
\begin{gathered}
\mathcal{N}^{\prime}=\frac{1}{2} \beta^{2}+\gamma^{\prime}, \mathcal{N}^{\prime \prime}=\gamma^{\prime \prime} \\
\mathcal{D}^{\prime}=\frac{1}{2} \mathcal{N}^{\prime 2}-\gamma^{2}+\zeta^{\prime}+\gamma^{\prime} \beta^{2}-4 \sum_{i=1}^{3} \gamma_{i}^{\prime} \beta^{i}, \\
\mathcal{D}^{\prime \prime}=\mathcal{N}^{\prime} \gamma^{\prime \prime}-\frac{1}{2} \gamma^{\prime \prime 2}-2 \gamma^{\prime} \gamma^{\prime \prime}+\varphi^{\prime \prime}+\psi^{\prime \prime}+\nu^{\prime \prime}+\gamma^{\prime \prime} \beta^{2}-4 \sum_{i=1}^{3} \gamma_{i}^{\prime \prime} \beta^{i} .
\end{gathered}
$$

Indeed, the internal forces resulting from $\gamma^{\prime \prime}$ are in general stronger than the external forces resulting from the potential $\gamma^{\prime}$. Nevertheless, in the equation ruling the motion of the center of gravity, the contributions of derivatives of $\gamma^{\prime \prime}$ cancel pairwise exactly. It is here that resides, conceptually, the origin of the cancellation principle in classical mechanics [20].

\section{Center of gravity, quasi-translational motions, size vs. distance}

The center of gravity is a geometric property of any body. In Newtonian mechanics, it represents the average location of the weight of an object, being defined as that point through which the resultant of the system of parallel forces formed by the weights of all the particles constituting the body passes, for all positions of the body. Therefore, the center of gravity represents the point in a body around which the resultant torque due to gravity forces vanishes. It is thus clear that in a uniform gravitational field the center of gravity is identical to the center of mass. We can completely describe the motion of any object through space in terms of the translation of the center of gravity of the object from one place to another, and the rotation of the object about its center of gravity if it is free to rotate. For a continuous system $S$ which, at time $t$, occupies the field $C$, the center of gravity of Newtonian theory is characterized by the equation $[20]$

$$
\Gamma-O=\frac{1}{m} \int_{C}(P-O) \mathrm{d} m,
$$

where $O$ is an arbitrary point for which we choose the origin of a Cartesian system Oxyz.

However, we stress that the definition (2.45) of center of gravity adopted throughout this paper differs from the one advocated in the modern literature, i.e., the point at which the Blanchet-Damour mass-dipole moment vanishes ${ }^{4}$.

\footnotetext{
${ }^{4}$ For a clear definition of mass-multipole moments see Refs. [29, 30].
} 
The Blanchet-Damour post-Newtonian mass-dipole moment $\mathcal{M}_{i}(t)(i=1,2,3)$ of a dynamical system can be written as $[29]$

$$
\mathcal{M}_{i}(t)=\int \mathrm{d}^{3} x x_{i} \sigma(x)-\frac{1}{c^{2}} \frac{\mathrm{d}}{\mathrm{d} t} \int \mathrm{d}^{3} x \sigma(x)^{j}\left(x_{i} x_{j}-\frac{1}{2} \delta_{i j} x^{2}\right)+\mathrm{O}\left(c^{-4}\right),
$$

where $\sigma_{\mu}(x)$ represent densities of mass and currents, i.e.,

$$
\begin{aligned}
\sigma(x) & =\frac{T^{00}+T_{i}^{i}}{c^{2}}, \\
\sigma(x)^{i} & =\frac{T^{0 i}}{c},
\end{aligned}
$$

$T^{\mu \nu}$ denoting the components of the stress-energy tensor in a global coordinate system. It is possible to demonstrate that $\mathcal{M}_{i}(t)$ satisfies the conservation law [29]

$$
\frac{\mathrm{d}^{2}}{\mathrm{~d} t^{2}} \mathcal{M}_{i}(t)=0
$$

since its first-order time derivative represents the conserved total linear momentum of the system. As is shown in Ref. [33], it is precisely the condition of vanishing Blanchet-Damour dipole moment that finally leads to the Einstein-Infeld-Hoffmann equations of motion for a system of mass monopoles.

At this stage, some further physical assumptions are here made that are indeed satisfied approximately by planetary motions in the solar system. They are as follows [20].

A1 The center of gravity $P_{G}$ of each body $C$ is substantial, i.e., it always adheres to the same material element. This implies that its motion will be characterized, as it occurs for any other material point $P$, by a Lagrangian $\mathcal{L}=\mathcal{N}+\mathcal{D}$, with the Einstein perturbation being given by the term $\mathcal{D}$. Furthermore, we shall assume that the center of gravity $P_{G}$ is always a center of gravitation. The latter condition means that $P_{G}$ is a point where the Newtonian attractions of material elements of the body ${ }^{5}$ (i.e., the internal forces) add up to zero.

A2 The body performs a quasi-translational motion. Indeed, in a translational motion, all points of the body have, at any instant $t$, the same vector speed, e.g., the speed $\vec{v}_{g}$ of the center of gravity. We can still regard as a translation every motion for which, defining

$$
|\triangle \vec{v}(t)| \equiv\left|\vec{v}_{P_{i}}(t)-\vec{v}_{P_{j}}(t)\right| \forall P_{i}, P_{j} \in C,
$$

one has always

$$
\frac{|\triangle \vec{v}|}{\left|\vec{v}_{g}\right|}<<1
$$

We need sufficiently small values of the ratio in (2.50), e.g., of order $10^{-2}$, so that one can neglect, as a quantity of order greater than 1, every product of the type

$$
\beta^{2} \frac{|\triangle \vec{v}|}{\left|\vec{v}_{g}\right|}, \gamma \frac{|\triangle \vec{v}|}{\left|\vec{v}_{g}\right|}
$$

This is precisely what happens for planetary motions. Their deformations are initially negligible and they behave, as a consequence, as essentially rigid bodies. Their motion is actually a composition of translation and rotation. However, for every point of the body, the speed resulting from rotation attains only a few percent of the common speed of translation. For example, in the case of the Earth, one has

$$
\frac{|\triangle \vec{v}|}{\left|\vec{v}_{g}\right|} \approx 3 \cdot 10^{-2}
$$

\footnotetext{
${ }^{5}$ Recall that the Newtonian potential $\gamma$ is bounded everywhere and it vanishes at infinity, hence there exists a point of minimum for $\gamma$ at which its gradient vanishes, which means that the force vanishes at this point.
} 
A3 On denoting by $d$ the maximal size of the body $C$, and by $R$ the minimal Euclidean distance $d_{E}$ between points of $C$ and points of the residual portion $S^{\prime}$ :

$$
R \equiv \min d_{E}\left(P_{j}(C), P_{k}\left(S^{\prime}\right)\right),
$$

the quantity $\left(\frac{d}{R}\right)^{2}$ is negligible. For the Sun-Earth system, one has indeed $\left(\frac{d}{R}\right)^{2} \approx 10^{-4}[20]$.

By virtue of $\mathbf{A} 1$, the gradient of the potential $\gamma^{\prime \prime}$ vanishes at the center of gravity, and hence $\gamma^{\prime \prime}$ behaves as a constant. Moreover, by virtue of A2, the dimensionless velocities $\beta^{i}$ defined in (2.25) are constant within the body $C$. On defining

$$
\gamma^{\prime \prime} \equiv \widetilde{\omega}=\text { constant }
$$

one finds from (2.26)-(2.30)

$$
\begin{gathered}
-\varphi^{\prime \prime}=\widetilde{\omega} \gamma^{\prime \prime}=\widetilde{\omega}^{2}, \\
\psi^{\prime \prime}=\frac{3}{2} \beta^{2} \gamma^{\prime \prime}=\frac{3}{2} \widetilde{\omega} \beta^{2}, \\
\gamma_{i}^{\prime \prime}=\beta^{i} \gamma^{\prime \prime}=\widetilde{\omega} \beta^{i}, \\
\nu^{\prime \prime}=\frac{1}{2} \frac{G_{N}}{c^{2}} \frac{\partial^{2}}{\partial\left(x^{0}\right)^{2}} \int_{C} \mu r \mathrm{~d} C=0, r=d_{E}\left(P_{j}, P_{G}\right) \forall P_{j} \in C .
\end{gathered}
$$

We stress that $\nu^{\prime \prime}$ vanishes because the integral in (2.56) is constant during the motion.

\section{E. The $\chi_{k}$ coefficients}

Hereafter we denote with $P_{k}$ the center of gravity of the body $C_{k}$, for all $k=0,1, \ldots, n-1$. We also denote with $l_{k}$ the gravitational radius of the $k$-th body having mass $m_{k}$ (we assume, on experimental ground, the equality of inertial and gravitational mass, and also of active and passive gravitational mass), i.e.,

$$
l_{k} \equiv \frac{G_{N} m_{k}}{c^{2}},
$$

which, in the solar system, does not exceed the $1.5 \mathrm{~km}$ for the gravitational radius of the Sun. The assumption A3 implies that the potential of the body $C_{k}$ acting on the center of gravity $P_{h}$ of $C_{h}$ is given, as if the mass of $C_{k}$ were completely concentrated at the center of gravity $P_{k}$, in the form $\frac{G_{N} m_{k}}{r\left(P_{k}, P_{h}\right)}$, where the denominator is the Euclidean distance $d_{E}\left(P_{k}, P_{h}\right)$. The dimensionless form of such a potential is obtained dividing by $c^{2}$, i.e.

$$
\frac{G_{N} m_{k}}{c^{2} r\left(P_{k}, P_{h}\right)}=\frac{l_{k}}{r\left(P_{k}, P_{h}\right)} \text {. }
$$

Now we need extra labels in the notation, since we are going to derive the Lagrangian in the form $\mathcal{N}+\mathcal{D}$ for each (celestial) body. For this purpose, following again Ref. [20], we shall denote by $\beta_{h}^{2}$ the square of the velocity of $P_{h}$, by $\left(\beta_{h}\right)_{i}$ the component along the axis $x^{i}$ of $\beta_{h}$, and by $\gamma_{h}^{\prime}$ the potential at $P_{h}$ resulting from all bodies $C_{k}$ described by an index $k \neq h$. This latter condition means that

$$
\gamma_{h}^{\prime}=\sum_{k=0}^{n-1}\left(1-\delta_{k, h}\right) \frac{l_{k}}{r\left(P_{k}, P_{h}\right)}
$$

with the understanding that the Kronecker $\delta$ plays the role of giving vanishing weight to the divergent term $\frac{l_{k}}{r\left(P_{k}, P_{k}\right)}$, which is therefore ruled out from the sum (sometimes this is expressed by the $\sum^{\prime}$ notation).

In light of (2.52), we can regard as being constant the integral

$$
\gamma_{h}^{\prime \prime}(P)=\frac{G_{N}}{c^{2}} \int_{C_{h}} \frac{\mu(Q)}{r(Q, P)} \mathrm{d} C_{h}=\widetilde{\omega}_{h}
$$


which is the potential of the body $C_{h}$ at any point $P$ of $C_{h}$ itself. The constant $\widetilde{\omega}_{h}$ is majorized by the ratio $\frac{\bar{l}}{\bar{r}}$, where $\left(\partial C_{h}\right.$ being the boundary of $\left.C_{h}\right)$

$$
\bar{r} \equiv \max d_{E}\left(P_{h}, \partial C_{h}\right)
$$

while $\bar{l} \equiv \frac{G_{N} \bar{m}}{c^{2}}, \bar{m}$ being the mass contained within a homogeneous sphere of density $\bar{\mu}$ and radius $\bar{r}$, having set

$$
\bar{\mu}=\sup (\mu) \text { within } C_{h}
$$

Equations (2.59)-(2.61) tell us that $\widetilde{\omega}$ is a quantity of first order, being close to a quantity proportional to a Newtonian potential. This simple property will be nicely exploited below.

Next, we consider an integration domain $S^{\prime}$ consisting of all bodies $C_{k}$ with the exception of the body $C_{h}$, in formulas

$$
S^{\prime} \equiv \cup_{k} C_{k}-C_{h}
$$

and, for points $Q \in S^{\prime}$ and $P \in C_{h}$, we consider the decomposition of the function $\varphi_{h}$ for the body $C_{h}$ in the form

$$
\varphi_{h}=\varphi_{h}^{\prime}+\varphi_{h}^{\prime \prime}
$$

where

$$
\varphi_{h}^{\prime}=-\frac{G_{N}}{c^{2}} \int_{S^{\prime}} \frac{\mu \gamma}{r(Q, P)} \mathrm{d} S^{\prime}
$$

In light of assumption A3, we can re-express (2.64) in the form

$$
\begin{aligned}
\varphi_{h}^{\prime} & =-\frac{G_{N}}{c^{2}} \sum_{k=0}^{n-1} \frac{\left(1-\delta_{k, h}\right)}{r\left(P_{k}, P\right)} \int_{C_{k}} \mu(Q) \gamma_{k}(Q) \mathrm{d} C_{k} \\
& =-\frac{G_{N}}{c^{2}} \sum_{k=0}^{n-1} \frac{\left(1-\delta_{k, h}\right)}{r\left(P_{k}, P\right)}\left(\sum_{j=0}^{n-1}\left(1-\delta_{j, k}\right) \frac{l_{j}}{r\left(P_{j}, P_{k}\right)} \int_{C_{k}} \mu(Q) \mathrm{d} C_{k}+\frac{G_{N}}{c^{2}} \int_{C_{k}} \mu(Q) \mathrm{d} C_{k} \int_{C_{k}} \frac{\mu\left(Q^{\prime}\right)}{r\left(Q, Q^{\prime}\right)} \mathrm{d} C_{k}\right) \\
& =-\sum_{k=0}^{n-1}\left(1-\delta_{k, h}\right) \frac{l_{k}}{r\left(P_{k}, P\right)} \sum_{j=0}^{n-1}\left(1-\delta_{j, k}\right) \frac{l_{j}}{r\left(P_{j}, P_{k}\right)}-\sum_{k=0}^{n-1}\left(1-\delta_{k, h}\right) \frac{l_{k} \chi_{k}}{r\left(P_{k}, P\right)}
\end{aligned}
$$

having defined [20]

$$
\chi_{k} \equiv \frac{1}{l_{k}}\left(\frac{G_{N}}{c^{2}}\right)^{2} \int_{C_{k}} \mu(Q) \mathrm{d} C_{k} \int_{C_{k}} \frac{\mu\left(Q^{\prime}\right)}{r\left(Q^{\prime}, Q\right)} \mathrm{d} C_{k}=\frac{G_{N}}{c^{2}} \widetilde{\omega}_{k}=\text { constant }
$$

and where each $\gamma_{k}$ potential in (2.65) has been split as in $(2.40)$, i.e., $\gamma_{k}^{\prime}(Q)+\gamma_{k}^{\prime \prime}(Q)$, and use of $(2.58)$ and $(2.59)$ has been made in order to express $\gamma_{k}^{\prime}(Q)$ and $\gamma_{k}^{\prime \prime}(Q)$, respectively. The existence of the constant coefficients $\chi_{k}$ is conceptually interesting, but their values are extremely small, because the integrals occurring in (2.66) are finite but are multiplied by the square of the ratio $\frac{G_{N}}{c^{2}}$.

\section{F. The effacement property}

At this stage, one can obtain the desired decomposition of the Lagrangian for the $h$-th body $C_{h}$ in the form [20]

$$
\mathcal{L}_{h}=\mathcal{N}_{h}+\mathcal{D}_{h}^{\prime}+\mathcal{D}_{h}^{\prime \prime}
$$

where $\mathcal{N}_{h}$ is the Newtonian term

$$
\mathcal{N}_{h}=\frac{1}{2}\left(\beta_{h}\right)^{2}+\gamma_{h}^{\prime}=\mathcal{N}_{h}^{\prime}
$$

while $\mathcal{D}_{h}^{\prime}$ is the pointlike Einstein perturbation

$$
\mathcal{D}_{h}^{\prime}=\frac{1}{2}\left(\mathcal{N}_{h}^{\prime}\right)^{2}-\left(\gamma_{h}^{\prime}\right)^{2}+\zeta_{h}^{\prime}+\gamma_{h}^{\prime}\left(\beta_{h}\right)^{2}-4 \sum_{i=1}^{3}\left(\Gamma_{h}^{\prime}\right)_{i}\left(\beta_{h}\right)_{i}
$$


$\left(\beta_{h}\right)_{i}$ denoting the $i$-th component of the velocity of the $h$-th body as we said before (2.58), and

$$
\left(\Gamma_{h}^{\prime}\right)_{i} \equiv \sum_{k=0}^{n-1}\left(1-\delta_{k, h}\right) \frac{l_{k}\left(\beta_{k}\right)_{i}}{r\left(P_{k}, P_{h}\right)}
$$

The expression (2.67) of the Lagrangian is completed by $\mathcal{D}_{h}^{\prime \prime}$, i.e. the Einstein perturbation resulting from the extension of bodies. Upon defining

$$
m \equiv \sum_{k=0}^{n-1} m_{k}, l \equiv \frac{G_{N} m}{c^{2}}, l_{k} \equiv \frac{G_{N} m_{k}}{c^{2}}, \lambda_{k} \equiv \frac{m_{k}}{m}=\frac{l_{k}}{l},
$$

the perturbation $\mathcal{D}_{h}^{\prime \prime}$ reads as

$$
\mathcal{D}_{h}^{\prime \prime}=-\widetilde{\omega}_{h}\left(\beta_{h}\right)^{2}-l \sum_{k=0}^{n-1}\left(1-\delta_{k, h}\right) \frac{\lambda_{k}\left(\chi_{k}+2 \widetilde{\omega}_{h}\right)}{r\left(P_{k}, P_{h}\right)} .
$$

We can now recall that Lagrangians differing by a multiplicative constant give rise to equivalent equations of motion. The simple and profound idea of Levi-Civita was to consider a first-order quantity $\sigma_{h}$, and to multiply $\mathcal{L}_{h}$ by $\left(1+\sigma_{h}\right)$. After doing this, one can try to choose $\sigma_{h}$ in such a way that the occurrence of the constant $\widetilde{\omega}_{h}$ gets exactly cancelled. This is indeed feasible because, up to higher order terms here negligible, one finds

$$
\left(1+\sigma_{h}\right) \mathcal{L}_{h} \sim\left(1+\sigma_{h}\right) \mathcal{N}_{h}+\mathcal{D}_{h}^{\prime}+\mathcal{D}_{h}^{\prime \prime}
$$

where, in particular,

$$
\left(1+\sigma_{h}\right) \mathcal{N}_{h}+\mathcal{D}_{h}^{\prime \prime}=\frac{1}{2}\left(\beta_{h}\right)^{2}+\left(\frac{1}{2} \sigma_{h}-\widetilde{\omega}_{h}\right)\left(\beta_{h}\right)^{2}+l \sum_{k=0}^{n-1}\left(1-\delta_{k, h}\right) \frac{\lambda_{k}\left[\left(1-\chi_{k}\right)+\left(\sigma_{h}-2 \widetilde{\omega}_{h}\right)\right]}{r\left(P_{k}, P_{h}\right)} .
$$

This formula suggests choosing

$$
\sigma_{h}=2 \widetilde{\omega}_{h},
$$

to achieve the desired cancellation. The result is also consistent with what we know already about the first-order nature of the constant $\widetilde{\omega}_{h}$. We can further define

$$
\Lambda_{k} \equiv \lambda_{k}\left(1-\chi_{k}\right),
$$

Note also that the pointlike Einstein perturbation $\mathcal{D}_{h}^{\prime}$ is still expressed in terms of the dimensionless $\lambda_{k}$ coefficients, but we can insert also therein the $\Lambda_{k}$ defined in (2.76), because

$$
\mathcal{D}_{h}^{\prime}\left(\lambda_{k}\right)=\mathcal{D}_{h}^{\prime}\left(\Lambda_{k}\right)+\text { higher }- \text { order terms. }
$$

One finds therefore that each (celestial) body is ruled by a pointlike Lagrangian $L_{h}$ where the Einstein perturbation is no longer split into pointlike plus finite-size part, and one can write [20]

$$
L_{h}=\mathcal{N}_{h}+\mathcal{D}_{h},
$$

where, having defined

$$
\begin{gathered}
\gamma_{h} \equiv l \sum_{k=0}^{n-1}\left(1-\delta_{k, h}\right) \frac{\Lambda_{k}}{r\left(P_{k}, P_{h}\right)} \\
\left(\Gamma_{h}\right)_{i} \equiv l \sum_{k=0}^{n-1}\left(1-\delta_{k, h}\right) \frac{\Lambda_{k}\left(\beta_{k}\right)_{i}}{r\left(P_{k}, P_{h}\right)}, \\
\zeta_{h} \equiv \varphi_{h}+\psi_{h}+\nu_{h},
\end{gathered}
$$




$$
\begin{gathered}
\varphi_{h} \equiv-l^{2} \sum_{k=0}^{n-1}\left[\left(1-\delta_{k, h}\right) \frac{\Lambda_{k}}{r\left(P_{k}, P_{h}\right)} \sum_{s=0}^{n-1}\left(1-\delta_{s, k}\right) \frac{\Lambda_{s}}{r\left(P_{s}, P_{k}\right)}\right] \\
\psi_{h} \equiv \frac{3}{2} l \sum_{k=0}^{n-1}\left(1-\delta_{k, h}\right) \frac{\Lambda_{k}\left(\beta_{k}\right)^{2}}{r\left(P_{k}, P_{h}\right)} \\
\nu_{h} \equiv \frac{1}{2} l \frac{\partial^{2}}{\partial\left(x^{0}\right)^{2}} \sum_{k=0}^{n-1}\left(1-\delta_{k, h}\right) \Lambda_{k} r\left(P_{k}, P_{h}\right),
\end{gathered}
$$

the Newtonian term takes the familiar form

$$
\mathcal{N}_{h} \equiv \frac{1}{2}\left(\beta_{h}\right)^{2}+\gamma_{h}
$$

while the Einstein perturbation is eventually expressed by the sum of functions

$$
\mathcal{D}_{h} \equiv \frac{1}{2}\left(\mathcal{N}_{h}\right)^{2}-\left(\gamma_{h}\right)^{2}+\zeta_{h}+\gamma_{h}\left(\beta_{h}\right)^{2}-4 \sum_{i=1}^{3}\left(\Gamma_{h}\right)_{i}\left(\beta_{h}\right)_{i} .
$$

Equations (2.78)-(2.86) lead to an accurate scheme for writing down and studying the equations of motion of each (celestial) body, and provide a precise statement of the cancellation principle in General Relativity: on going from Newtonian to relativistic celestial mechanics, the effects of extension and internal structure of bodies are encoded in the family of $\Lambda_{k}$ parameters, Eq. (2.76), which differ only by a tiny amount (see Eq. (2.66)) from the dimensionless mass ratios $\frac{m_{k}}{m}$ of Eq. (2.71). Thus, the effects of finite extension of bodies get eventually dissolved neatly, and it is as if we were dealing with material points which do not affect at all their center of gravity. This holds approximately for the solar system.

As is stressed in Ref. [31], a more rigorous proof of such a cancellation principle can be found in Ref. [19], to which we refer the reader interested in a broader understanding.

\section{G. Multipolar expansion}

We have already stressed that, although the Levi-Civita analysis has been nowadays superseded in many circumstances, it still represents a useful tool to investigate relativistic dynamics involving planets of the Solar System, the predicted equations being highly complicated, as we will show below. Anyway, the multipolar-post-Minkowskian approach, developed in Ref. [27] in order to construct the most general past-stationary and past-asymptotically Minkowskian formal solution of the linearized harmonic vacuum Einstein equations, can be applied also to our Eqs. (2.78)-(2.86). Within such a framework, a formal series is introduced in powers of $G_{N}$ involving the spacetime metric in the form

$$
\sqrt{-g} g^{\mu \nu}=\eta^{\mu \nu}+G_{N} h_{1}^{\mu \nu}+\left(G_{N}\right)^{2} h_{2}^{\mu \nu}+\cdots+\left(G_{N}\right)^{n} h_{n}^{\mu \nu}+\ldots,
$$

$\eta^{\mu \nu}$ being the flat metric. Each term $h_{n}^{\mu \nu}\left(x^{\alpha}\right)$ is supposed to admit a (finite) multipolar expansion associated with the $O(3)$ group of spatial rotations (leaving the quantities $\underline{r}=\sqrt{\sum_{i=1}^{3} x_{i}^{2}}$ and $t=x^{0} / c$ invariant) expressed by the concise relation $[27]$

$$
h_{n}^{\mu \nu}\left(x^{\alpha}\right)=\sum_{l=0}^{l_{\max }} h_{n L}^{\mu \nu}(\underline{r}, t) \hat{n}^{L}(\theta, \phi),
$$

where $l_{\max }$ represents some maximum value of $l$ depending on $n, L$ is a multi-index such that $n^{L}=n^{i_{1}} n^{i_{2}} \ldots n^{i_{l}}$ with $n^{i}=x^{i} / \underline{r}(i=1,2,3)$, and $\hat{n}^{L}(\theta, \phi)$ denotes the symmetric-trace-free part of $n^{L}$ (recall that the set of all symmetrictrace-free Cartesian tensors of rank $l$ generates an irreducible representation of weight $l$ and dimension $2 l+1$ of the special orthogonal group $S O(3)$ ). Moreover, it is possible to show that the sum occurring on the right-hand side of (2.88) is equivalent to a finite expansion in the usual spherical harmonics $Y_{l}^{m}(\theta, \phi)$ of the 2 -sphere [27]. However, the dynamical equations occurring within such a pattern still remain very complicated to handle. 


\section{SUN-EARTH, SUN-EARTH-MOON AND $N$-BODY DYNAMICS}

Driven by the concepts outlined in the previous sections, we now aim at investigating the system consisting of the Sun, Earth and as many additional celestial bodies as possible by means of the Lagrangian (2.78). An important comment should be made at this stage. In fact, in the most general case, acceleration terms appear in (2.78). However, by bearing in mind that $\chi_{k} \ll 1$ and $\lambda_{k}=$ constant (cf. Eqs. (2.66) and (2.71)), it easily follows that

$$
\nu_{h}=0, \quad \forall h,
$$

since the Euclidean distance $r\left(P_{k}, P_{h}\right)$ occurring in Eq. (2.84) depends on time only implicitly, through the coordinates $x^{i}(t)$, i.e.,

$$
\frac{\partial}{\partial t} r\left(P_{k}, P_{h}\right)=\frac{\partial}{\partial t} r\left(x^{1}(t), x^{2}(t), x^{3}(t)\right)=0 .
$$

In other words, under our assumption the Lagrangian (2.78) turns out to be a function of the Euclidean coordinates $x^{1}(t), x^{2}(t), x^{3}(t)$ and their first time derivatives only. Moreover, Eq. (3.1) is valid also in the more general case of a Lagrangian function depending explicitly on the time variable $t$, since the value of the integral

$$
\int_{\mathrm{D}} \mu r \mathrm{~d} C
$$

evaluated in the region D made up of all those spatial points which are very distant from $P_{h}$ reduces nearly to zero because it turns out to be of order $\left(\frac{d}{R}\right)^{2}$, which, according to hypothesis A3, represents a negligible quantity.

As a first step, we have analysed the system made up of just two bodies by considering the case of one celestial body orbiting a fixed massive object (i.e., the Sun). We have found that perihelion shift predicted by the Levi-Civita Lagrangian is in accordance with the well-known results expected within the usual 1PN picture of general relativity. In fact by employing the latter approximation, the orbit of the revolving body (in the equatorial plane $\theta=\pi / 2$ ) is described by the well-known relations [93]

$$
r(\phi)=\frac{\left(1-e^{2}\right) a}{1+e \cos \left[\left(1-\delta \phi_{0} / 2 \pi\right) \phi\right]},
$$

and

$$
\begin{aligned}
& x=e a+r(\phi) \cos \phi, \\
& y=r(\phi) \sin \phi,
\end{aligned}
$$

with

$$
\delta \phi_{0}=\frac{6 \pi G_{N} M}{c^{2} a\left(1-e^{2}\right)},
$$

$e$ being the eccentricity of the orbit of the orbiting body, $a$ the semi-major axis and $M$ the mass of the massive object. As demonstrated in Figs. 1 and 2, the outcomes achieved within the Levi-Civita framework are in agreement with the ones expected through the 1PN approximation method, witnessing that Levi-Civita actually made a mistake in Ref. [20] when concluding that his pattern predicts a more pronounced shift of the perihelion in the orbit of the revolving body. This point is in accordance with the analysis of Ref. [31].

Moreover, the Newtonian relations expressing the eccentricity $e$ of the orbit as a function of the initial velocity $v_{0}$ of the revolving body and its distance $\mathcal{R}$ from the massive one according to

$$
\begin{array}{ll}
v_{0}=\sqrt{\frac{G_{N} M}{\mathcal{R}}}, & \text { circular orbit, } \\
0<v_{0}<\sqrt{\frac{2 G_{N} M}{\mathcal{R}}},\left(v_{0} \neq \sqrt{\frac{G_{N} M}{\mathcal{R}}}\right), & \text { elliptic orbit, } \\
v_{0}=\sqrt{\frac{2 G_{N} M}{\mathcal{R}}}, & \text { parabolic orbit, } \\
v_{0}>\sqrt{\frac{2 G_{N} M}{\mathcal{R}}}, & \text { hyperbolic orbit, }
\end{array}
$$




$$
\begin{aligned}
& h_{1}(x, y, \dot{x}, \dot{y})=\frac{l m_{E} \Lambda_{S}\left[-4 c^{2} l \Lambda_{E} r x-2 c^{2} l \Lambda_{S} r x+r^{2}\left(-6 y \dot{x} \dot{y}+2 c^{2} x-3 x \dot{x}^{2}+3 x \dot{y}^{2}\right)\right]}{2 r^{5}}, \\
& h_{2}(x, y, \dot{x}, \dot{y})=\frac{l m_{E} \Lambda_{S}\left[-4 c^{2} l \Lambda_{E} r y-2 c^{2} l \Lambda_{S} r y+r^{2}\left(-6 x \dot{x} \dot{y}+2 c^{2} y+3 x \dot{x}^{2}-3 y \dot{y}^{2}\right)\right]}{2 r^{5}} .
\end{aligned}
$$

Note that it is possible to obtain $h_{2}(x, y, \dot{x}, \dot{y})$ starting from $h_{1}(x, y, \dot{x}, \dot{y})$ (or vice-versa) by setting

$$
\begin{aligned}
& x \longleftrightarrow y, \\
& \dot{x}^{2} \rightarrow-\dot{x}^{2}, \\
& \dot{y}^{2} \rightarrow-\dot{y}^{2},
\end{aligned}
$$

whereas the terms linear in the velocities have the freedom to change according to

$$
\begin{aligned}
& \dot{x} \rightarrow \pm \dot{x}, \\
& \dot{y} \rightarrow \pm \dot{y} .
\end{aligned}
$$

\section{B. Sun-Earth-Moon system}

If we analyse the Sun-Earth-Moon system, the Lagrangian for the Moon becomes

$$
m_{M} c^{2} \mathcal{L}_{M}(x, y, \dot{x}, \dot{y})=m_{M} c^{2}\left(\mathcal{N}_{M}+\mathcal{D}_{M}\right),
$$

$m_{M}$ being the mass of the Moon, while

$$
\begin{gathered}
\gamma_{M}=\frac{l_{S}}{r}+\frac{l_{E}}{r_{E M}}, \\
\varphi_{M}=-l^{2}\left[\frac{\Lambda_{S}}{r}\left(\frac{\Lambda_{E}}{r_{E}}+\frac{\Lambda_{M}}{r}\right)+\frac{\Lambda_{E}}{r_{E M}}\left(\frac{\Lambda_{M}}{r_{E M}}+\frac{\Lambda_{S}}{r_{E}}\right)\right],
\end{gathered}
$$

where now $r=r(t)=\sqrt{x^{2}(t)+y^{2}(t)}$ denotes the distance of the Moon from the Sun, $r_{E M}=\mid \mathbf{r}-$ $\mathbf{r}_{E} \mid=\sqrt{\left(x_{E}(t)-x(t)\right)^{2}+\left(y_{E}(t)-y(t)\right)^{2}}$ represents the distance between the Earth, having coordinates $\mathbf{r}_{E}(t)=$ $\left(x_{E}(t), y_{E}(t)\right)$, and the Moon, whereas $r_{E}$ is the distance between the Sun and the Earth. The resulting EulerLagrange equations, giving rise to Fig. 3, assume the form

$$
\left\{\begin{array}{l}
\tilde{f}\left(x, y, x_{E}, y_{E}, \dot{x}, \dot{y}\right) \ddot{x}+\tilde{g}(\dot{x}, \dot{y}) \ddot{y}+\tilde{h}_{1}\left(x, y, x_{E}, y_{E}, \dot{x}, \dot{y}\right)=0, \\
\tilde{g}(\dot{x}, \dot{y}) \ddot{x}+\tilde{f}\left(x, y, x_{E}, y_{E}, \frac{\dot{x}}{\sqrt{3}}, \sqrt{3} \dot{y}\right) \ddot{y}+\tilde{h}_{2}\left(x, y, x_{E}, y_{E}, \dot{x}, \dot{y}\right)=0
\end{array}\right.
$$

with

$$
\begin{gathered}
\tilde{f}\left(x, y, x_{E}, y_{E}, \dot{x}, \dot{y}\right)=\frac{m_{M}}{2 c^{2}}\left(2 c^{2}+\frac{6 c^{2} l_{S}}{r}+\frac{6 c^{2} l_{E}}{r_{E M}}+3 \dot{x}^{2}+\dot{y}^{2}\right), \\
\tilde{g}(\dot{x}, \dot{y})=\frac{m_{M} \dot{x} \dot{y}}{c^{2}}
\end{gathered}
$$




$$
\begin{aligned}
\tilde{h}_{1}\left(x, y, x_{E}, y_{E}, \dot{x}, \dot{y}\right) & =\frac{1}{2} m_{M}\left\{\frac { 1 } { r _ { E M } ^ { 3 } } \left(\frac{2 c^{2} l_{E}^{2} x_{E}}{r_{E M}}+\frac{4 c^{2} l^{2} \Lambda_{E} \Lambda_{M} x_{E}}{r_{E M}}+2 c^{2} l_{E} x_{E}+2 c^{2} l^{2} \Lambda_{E} \Lambda_{S}\left(x_{E} / r_{E}\right)\right.\right. \\
& \left.+\frac{2 c^{2} l_{E} l_{S} x_{E}}{r}+3 l_{E} x_{E} \dot{x}^{2}+6 l_{E} y_{E} \dot{x} \dot{y}-3 l_{E} x_{E} \dot{y}^{2}\right)+6 y \dot{x}\left[\frac{l_{E} \omega_{E} x_{E}}{r_{E M}^{3}}-\dot{y}\left(\frac{l_{S}}{r^{3}}+\frac{l_{E}}{r_{E M}^{3}}\right)\right] \\
& +x\left[2 c ^ { 2 } \left(-\frac{l_{S}^{2}}{r^{4}}-\frac{2 l^{2} \Lambda_{M} \Lambda_{S}}{r^{4}}-\frac{l^{2} \Lambda_{E} \Lambda_{S}}{r_{E} r^{3}}-\frac{l_{E}^{2}}{r_{E M}^{4}}-\frac{2 l^{2} \Lambda_{E} \Lambda_{M}}{r_{E M}^{4}}+\frac{l_{E}}{r_{E M}^{3}}-\frac{l^{2} \Lambda_{E} \Lambda_{S}}{r_{E} r_{E M}^{3}}\right.\right. \\
& \left.\left.\left.+\frac{l_{S}}{r^{3}}+\frac{l_{S} l_{E}}{r r_{E M}^{3}}-\frac{l_{S} l_{E}}{r^{3} r_{E M}}\right)-\frac{6 l_{E} \omega_{E} y \dot{x}}{r_{E M}^{3}}+3\left(\dot{y}^{2}-\dot{x}^{2}\right)\left(\frac{l_{S}}{r^{3}}+\frac{l_{E}}{r_{E M}^{3}}\right)\right]\right\},
\end{aligned}
$$

$\omega_{E}$ being the classical pulsation associated to the motion of the Earth around the Sun and, likewise the two-body case,

$$
\tilde{h}_{1}\left(x, y, x_{E}, y_{E}, \dot{x}, \dot{y}\right) \longleftrightarrow \tilde{h}_{2}\left(x, y, x_{E}, y_{E}, \dot{x}, \dot{y}\right)
$$

if

$$
\begin{aligned}
& x \longleftrightarrow y, \\
& x_{E} \longleftrightarrow y_{E}, \\
& \dot{x} \rightarrow-\dot{x}, \\
& \dot{y} \rightarrow-\dot{y}, \\
& \dot{x}^{2} \rightarrow-\dot{x}^{2}, \\
& \dot{y}^{2} \rightarrow-\dot{y}^{2} .
\end{aligned}
$$

C. Case of the $h$-th body immersed in the gravitational field produced by the other $(n-1)$ bodies

Driven by the features of the above analysis, it is possible to infer the presence of a recursive scheme according to which the Lagrange equations resulting from Eqs. (2.78) and (3.9), and describing the motion of the $h$-th body immersed in the gravitational field produced by the other $n-1$ bodies, are given by

$$
\left\{\begin{array}{l}
\mathcal{F}\left(x_{h}, y_{h}, x_{k}, y_{k}, \dot{x}_{h}, \dot{y}_{h}\right) \ddot{x}_{h}+\mathcal{G}\left(\dot{x}_{h}, \dot{y}_{h}\right) \ddot{y}_{h}+\mathcal{H}_{1}\left(x_{h}, y_{h}, x_{k}, y_{k}, \dot{x}_{h}, \dot{y}_{h}\right)=0, \\
\mathcal{G}\left(\dot{x}_{h}, \dot{y}_{h}\right) \ddot{x}_{h}+\mathcal{F}\left(x_{h}, y_{h}, x_{k}, y_{k}, \frac{\dot{x}_{h}}{\sqrt{3}}, \sqrt{3} \dot{y}_{h}\right) \ddot{y}_{h}+\mathcal{H}_{2}\left(x_{h}, y_{h}, x_{k}, y_{k}, \dot{x}_{h}, \dot{y}_{h}\right)=0, \quad(k=0,1, \ldots, n-1),(k \neq h),
\end{array}\right.
$$

possessing the property

$$
\mathcal{H}_{1}\left(x_{h}, y_{h}, x_{k}, y_{k}, \dot{x}_{h}, \dot{y}_{h}\right) \longleftrightarrow \mathcal{H}_{2}\left(x_{h}, y_{h}, x_{k}, y_{k}, \dot{x}_{h}, \dot{y}_{h}\right)
$$

when

$$
\begin{aligned}
& x_{h} \longleftrightarrow y_{h}, \\
& x_{k} \longleftrightarrow y_{k}, \\
& \dot{x}_{h} \rightarrow-\dot{x}_{h}, \\
& \dot{y}_{h} \rightarrow-\dot{y}_{h}, \\
& \dot{x}_{h}^{2} \rightarrow-\dot{x}_{h}^{2}, \\
& \dot{y}_{h}^{2} \rightarrow-\dot{y}_{h}^{2} .
\end{aligned}
$$

\section{LINEAR SYSTEM OF ORDINARY DIFFERENTIAL EQUATIONS ASSOCIATED WITH THE NONLINEAR EQUATIONS OF MOTION}

The expressions describing the relativistic motion of a massive object we have derived in the previous section (cf. Eqs. (3.15), (3.25), and (3.31)) clearly represent a coupled second-order system of nonlinear ordinary differential 
equations. All the coefficients occurring in these equations can be seen as smooth and differentiable functions on $\mathbb{R}^{2}$ since the distance scales occurring in our framework prevent the bodies from colliding. This means that our model cannot be employed to investigate the binary systems analysed in relativistic astrophysics [42, 44, 50, 52, 54, 55, 64-67], or those systems made up of comets or asteroids hitting a planet or other celestial bodies.

We now aim at showing that it is possible to map such systems into a second-order system of linear ordinary differential equations by applying the time derivative operator $\frac{\mathrm{d}}{\mathrm{d} t}$ four times to the original equations. The resulting expressions contain the fourth-order time derivative of the functions $x(t)$ and $y(t)$ as their unknowns. Consider the system (3.31) written in matrix form

$$
\mathcal{A}\left[\begin{array}{c}
\ddot{x}_{h} \\
\ddot{y}_{h}
\end{array}\right]+\left[\begin{array}{c}
\mathcal{H}_{1}\left(x_{h}, y_{h}, x_{k}, y_{k}, \dot{x}_{h}, \dot{y}_{h}\right) \\
\mathcal{H}_{2}\left(x_{h}, y_{h}, x_{k}, y_{k}, \dot{x}_{h}, \dot{y}_{h}\right)
\end{array}\right]=0
$$

where

$$
\mathcal{A}=\left[\begin{array}{cc}
\mathcal{F}\left(x_{h}, y_{h}, x_{k}, y_{k}, \dot{x}_{h}, \dot{y}_{h}\right) & \mathcal{G}\left(\dot{x}_{h}, \dot{y}_{h}\right) \\
\mathcal{G}\left(\dot{x}_{h}, \dot{y}_{h}\right) & \mathcal{F}\left(x_{h}, y_{h}, x_{k}, y_{k}, \frac{\dot{x}_{h}}{\sqrt{3}}, \sqrt{3} \dot{y}_{h}\right)
\end{array}\right]
$$

Thus, we can write Eq. (3.31) as (hereafter the convention on the summation over repeated indices is employed)

$$
\mathcal{A}_{i r}\left(x_{j}, \dot{x}_{j}\right) \ddot{x}_{r}+\mathcal{H}_{i}\left(x_{j}, \dot{x}_{j}\right)=0, \quad(i=1,2) .
$$

Bearing in mind the obvious relations

$$
\begin{gathered}
\frac{\mathrm{d}}{\mathrm{d} t} \mathcal{A}_{i r}=\dot{\mathcal{A}}_{i r}=\frac{\partial \mathcal{A}_{i r}}{\partial x_{j}} \dot{x}_{j}+\frac{\partial \mathcal{A}_{i r}}{\partial \dot{x}_{j}} \ddot{x}_{j} \\
\frac{\mathrm{d}}{\mathrm{d} t} \mathcal{H}_{i}=\dot{\mathcal{H}}_{i}=\frac{\partial \mathcal{H}_{i}}{\partial x_{j}} \dot{x}_{j}+\frac{\partial \mathcal{H}_{i}}{\partial \dot{x}_{j}} \ddot{x}_{j}
\end{gathered}
$$

the first-order time derivative of (4.3) gives

$$
\mathcal{A}_{i r} \frac{\mathrm{d}^{2}}{\mathrm{~d} t^{2}} \dot{x}_{r}+\left(\frac{\partial \mathcal{A}_{i r}}{\partial x_{j}} \dot{x}_{j}+\frac{\partial \mathcal{A}_{i r}}{\partial \dot{x}_{j}} \ddot{x}_{j}\right) \frac{\mathrm{d}}{\mathrm{d} t} \dot{x}_{r}+\frac{\partial \mathcal{H}_{i}}{\partial x_{j}} \dot{x}_{j}+\frac{\partial \mathcal{H}_{i}}{\partial \dot{x}_{j}} \ddot{x}_{j}=0, \quad(i=1,2),
$$

which represents a nonlinear system of differential equations whose solutions are given by the functions $\dot{x}_{r}(r=1,2)$. The second-order time derivative of (4.3) leads to the system

$$
\begin{aligned}
& \mathcal{A}_{i r} \frac{\mathrm{d}^{2}}{\mathrm{~d} t^{2}} \ddot{x}_{r}+2\left(\frac{\partial \mathcal{A}_{i r}}{\partial x_{j}} \dot{x}_{j}+\frac{\partial \mathcal{A}_{i r}}{\partial \dot{x}_{j}} \ddot{x}_{j}\right) \frac{\mathrm{d}}{\mathrm{d} t} \ddot{x}_{r}+\ddot{x}_{r} \frac{\partial \mathcal{A}_{i r}}{\partial \dot{x}_{k}} \frac{\mathrm{d}}{\mathrm{d} t} \ddot{x}_{k}+\frac{\partial \mathcal{H}_{i}}{\partial \dot{x}_{k}} \frac{\mathrm{d}}{\mathrm{d} t} \ddot{x}_{k}+\left(\frac{\partial^{2} \mathcal{A}_{i r}}{\partial x_{l} \partial \dot{x}_{k}} \dot{x}_{l}+2 \frac{\partial^{2} \mathcal{A}_{i r}}{\partial \dot{x}_{l} \partial \ddot{x}_{l}} \dot{x}_{k}\right. \\
& \left.+\frac{\partial \mathcal{A}_{i r}}{\partial x_{k}} \ddot{x}_{k}+\frac{\partial^{2} \mathcal{A}_{i r}}{\partial \dot{x}_{l} \partial \dot{x}_{k}} \ddot{x}_{l} \ddot{x}_{k}\right) \ddot{x}_{r}+\frac{\partial^{2} \mathcal{H}_{i}}{\partial x_{l} \partial x_{k}} \dot{x}_{l} \dot{x}_{k}+2 \frac{\partial^{2} \mathcal{H}_{i}}{\partial \dot{x}_{l} \partial x_{k}} \ddot{x}_{l} \dot{x}_{k}+\frac{\partial \mathcal{H}_{i}}{\partial x_{k}} \ddot{x}_{k}+\frac{\partial^{2} \mathcal{H}_{i}}{\partial \dot{x}_{l} \partial \dot{x}_{k}} \ddot{x}_{l} \ddot{x}_{k}=0, \quad(i=1,2),
\end{aligned}
$$

still representing a nonlinear set of differential equations in the unknowns $\ddot{x}_{r}$. However, by patiently applying the time derivative operator four times to Eq. (4.3) (see Appendix A for details) the fourth time derivatives $x_{r}^{(4)}$ are found to solve a linear system of ordinary differential equations of the form

$$
\begin{aligned}
& \mathcal{A}_{i r} \frac{\mathrm{d}^{2}}{\mathrm{~d} t^{2}} x_{r}^{(4)}+4\left(\frac{\partial \mathcal{A}_{i r}}{\partial x_{j}} \dot{x}_{j}+\frac{\partial \mathcal{A}_{i r}}{\partial \dot{x}_{j}} \ddot{x}_{j}\right) \frac{\mathrm{d}}{\mathrm{d} t} x_{r}^{(4)}+\ddot{x}_{r}\left(\frac{\partial \mathcal{A}_{i r}}{\partial \dot{x}_{k}}\right) \frac{\mathrm{d}}{\mathrm{d} t} x_{k}^{(4)}+\left(\frac{\partial \mathcal{H}_{i}}{\partial \dot{x}_{k}}\right) \frac{\mathrm{d}}{\mathrm{d} t} x_{k}^{(4)}+F_{i}\left(x_{p}, \dot{x}_{p}, \ldots, x_{p}^{(4)}\right)=0, \\
& (i, p=1,2) .
\end{aligned}
$$

The form of $F_{i}\left(x_{p}, \dot{x}_{p}, \ldots, x_{p}^{(4)}\right)$ can be read from Eq. (A3), where it is explicitly shown that this term does not depend on time derivatives of $x_{p}$ having an order higher than the fourth and that its dependence on $x_{p}^{(4)}$ turns out to 
be linear. Therefore, we have obtained the original result according to which the differential equations describing the motion of a body in our Solar System within the first post-Newtonian approximation can be put in linear form if the fourth time derivative of the original equation is employed. In our analysis, we have been inspired by the work in Ref. [95], where the author differentiated (see below) a system of nonlinear partial differential equations to arrive at their solution. More precisely, starting from a system of $n$ nonlinear second-order hyperbolic partial differential equations of the form

$$
A^{\lambda \mu} \frac{\partial^{2} w_{s}}{\partial x^{\lambda} \partial x^{\mu}}+f_{s}=0, \quad s=1,2, \ldots, n, \quad \lambda, \mu=1,2,3,4,
$$

the coefficients $A^{\lambda \mu}$ and $f_{s}$ depending in a nonlinear way on the four variables $x^{\alpha}$, the unknown functions $w_{s}$, and their first time derivatives $\frac{\partial w_{s}}{\partial x^{\alpha}}$, in Ref. [95] it is shown that by applying five times of the derivative operator with respect to any whatsoever variable $x^{\alpha}$ leads to the linear system

$$
A^{\lambda \mu} \frac{\partial^{2} U_{S}}{\partial x^{\lambda} \partial x^{\mu}}+\frac{\partial U_{S}}{\partial x^{\lambda}}+F_{S}=0, \quad S=1,2, \ldots, N, \quad \lambda, \mu=1,2,3,4,{ }^{6}
$$

where $U_{S}$ represents the partial derivatives of order five of $w_{s}$ and $F_{S}$ is a function of the four variables $x^{\alpha}$, the unknown functions $w_{s}$, and of their partial derivatives up to the fifth order included, but not of the derivatives of higher order. In particular, when this framework is applied to Einstein's field equations (which represent a system of $n=10$ quasilinear partial differential equations) it suffices to derive them four times in order to obtain a linear system, where the unknowns are given by the fourth derivatives of the metric tensor components [95].

Having obtained a linear system of differential equations of second order associated to (3.31), we might in principle exploit their reduction to canonical form (Appendix B) and the rich qualitative theory [96] of such equations. In fact, as we have seen in Sec. III, our model predicts the presence of periodic solutions (see for example Figs. 1, 2, and 3) in simple cases, and hence the qualitative theory just mentioned and the encouraging evidence for simple (but nontrivial) two-body systems suggest undertaking the much harder analysis of $N$-body systems.

\section{QUANTUM EFFECTS ON LAGRANGIAN POINTS REVISITED}

The work in Secs. II and III puts on firm ground the investigations initiated in Refs. [1-4]. In other words, the motion of celestial bodies in the solar system can be studied by employing a Lagrangian that is almost independent of the internal structure [20]. Within Einstein's theory, the effacement property of Newtonian theory is still valid at the first post-Newtonian approximation, because (Sec. II) all large, direct self-action effects cancel each other or contribute terms in the equations of motion which can be removed, so that the final equations can be written in terms of only some centers of mass and some effective masses [20,28, 31]. But then, to the extent that the effective-gravity prescriptions are correct, it becomes legitimate to consider Newtonian potential terms among large masses in the Lagrangian used to derive geodesic motion of planets and other bodies, and insert therein the quantum modifications worked out in Refs. [5, 6, 11-13].

In particular, the work of Ref. [4] has studied in detail how quantum corrections on the relativistic position of the Earth-Moon Lagrangian points can be evaluated. In fact the effective gravity picture modifies the Newtonian potential among bodies of masses $m_{A}$ and $m_{B}$ in the low-energy/long-distance domain through the asymptotic expansion

$$
\begin{aligned}
V_{E}(r) & \sim-\frac{G_{N} m_{A} m_{B}}{r}\left[1+\left(\kappa_{1} \frac{\left(l_{A}+l_{B}\right)}{r}+\kappa_{2} \frac{\left(l_{P}\right)^{2}}{r^{2}}+\mathrm{O}\left(G_{N}^{2}\right)\right)\right] \\
\Longrightarrow & \frac{V_{E}(r)}{c^{2} m_{B}} \sim-\frac{l_{A}}{r}\left[1+\left(\kappa_{1} \frac{\left(l_{A}+l_{B}\right)}{r}+\kappa_{2} \frac{\left(l_{P}\right)^{2}}{r^{2}}+\mathrm{O}\left(G_{N}^{2}\right)\right)\right],
\end{aligned}
$$

$l_{A}$ and $l_{B}$ being the gravitational radii of the bodies and $l_{P}$ the Planck length, whereas $\kappa_{1}$ and $\kappa_{2}$ represent constants ${ }^{7}$ resulting from the calculation of Feynman diagrams involved in the particular definition adopted for the potential:

\footnotetext{
${ }^{6} N$ represents the product by $n$ of the number of partial derivatives of order five of a function of four variables.

${ }^{7}$ In this conceptual scheme, physical phenomena are described of course in a classical way on large distances, but the precise values of some coefficients depend on the underlying quantum theory. Thus, the dimensionless $\kappa_{1}$ parameter is the effective-gravity weight of the purely classical term $\frac{\left(l_{A}+l_{B}\right)}{r}$.
} 
one-particle reducible, scattering or bound-states (see Tab. I). The term $\kappa_{1} \frac{\left(l_{A}+l_{B}\right)}{r}$ in $(5.1)$ refers to a post-Newtonian correction to the classical potential, whereas $\kappa_{2} \frac{\left(l_{P}\right)^{2}}{r^{2}}$ represents a fully quantum term, depending on the square of the Planck length.

TABLE I: The values assumed by $\kappa_{1}$ and $\kappa_{2}$ in the three different potentials.

\begin{tabular}{|c|c|c|c|}
\hline$\kappa_{i}$ & one-particle reducible & scattering & bound-states \\
\hline$\kappa_{1}$ & -1 & 3 & $-\frac{1}{2}$ \\
\hline$\kappa_{2}$ & $-\frac{167}{30 \pi}$ & $\frac{41}{10 \pi}$ & $\frac{41}{10 \pi}$ \\
\hline
\end{tabular}

In Ref. [4] we have proposed a framework where the aforementioned quantum corrections to Lagrangian points can be obtained by constructing a map whose form is inspired by the pattern enlightened in Eq. (5.1). In particular, we have applied the map

$$
\left(U_{\alpha}, U_{\beta}\right) \rightarrow\left(V_{\alpha}, V_{\beta}\right)
$$

with

$$
\begin{gathered}
U_{\alpha}(r) \equiv \frac{l_{\alpha}}{r}=U_{\alpha}, \\
U_{\beta}(s) \equiv \frac{l_{\beta}}{s}=U_{\beta}, \\
V_{\alpha}(r) \sim\left[1+\kappa_{2} \frac{\left(l_{P}\right)^{2}}{r^{2}}\right] U_{\alpha}(r)+\kappa_{1}\left(U_{\alpha}(r)\right)^{2}+\mathrm{O}\left(G_{N}^{3}\right), \\
V_{\beta}(s) \sim\left[1+\kappa_{2} \frac{\left(l_{P}\right)^{2}}{s^{2}}\right] U_{\beta}(s)+\kappa_{1}\left(U_{\beta}(s)\right)^{2}+\mathrm{O}\left(G_{N}^{3}\right),
\end{gathered}
$$

to the Lagrangian describing the motion of the planetoid in the gravitational field generated by the Earth and the Moon, which, upon adopting the set of coordinates $(c t, \xi, \eta, \zeta)$ ( $c$ being the speed of light) reads as

$$
L=\frac{1}{2} \sum_{\mu, \nu=0}^{3} g_{\mu \nu} \frac{\mathrm{d} x^{\mu}}{\mathrm{d} t} \frac{\mathrm{d} x^{\nu}}{\mathrm{d} t},
$$

where

$$
\begin{aligned}
& g_{00}=1-2 \frac{l_{\alpha}}{r}-2 \frac{l_{\beta}}{s}-\frac{\Omega^{2}}{c^{2}}\left(\xi^{2}+\eta^{2}\right)+2\left[\left(\frac{l_{\alpha}}{r}\right)^{2}+\left(\frac{l_{\beta}}{s}\right)^{2}\right] \\
&-2 \frac{\left(l_{\alpha}+l_{\beta}\right)}{R^{3}}\left(\frac{l_{\alpha}}{r}+\frac{l_{\beta}}{s}\right)\left(\xi^{2}+\eta^{2}\right)+4 \frac{l_{\alpha}}{r} \frac{l_{\beta}}{s} \\
&+ \frac{(2-\rho)}{(1+\rho)} \frac{l_{\alpha}}{r} \frac{l_{\beta}}{R}+\frac{(2 \rho-1)}{(1+\rho)} \frac{l_{\beta}}{s} \frac{l_{\alpha}}{R}-7 \frac{\xi}{R^{2}}\left(\frac{l_{\alpha}}{r} l_{\beta}-\frac{l_{\beta}}{s} l_{\alpha}\right) \\
&+(1+\rho)^{-1} \frac{\eta^{2}}{R}\left[\rho\left(\frac{l_{\alpha}}{r}\right)^{3} \frac{l_{\beta}}{\left(l_{\alpha}\right)^{2}}+\left(\frac{l_{\beta}}{s}\right)^{3} \frac{l_{\alpha}}{\left(l_{\beta}\right)^{2}}\right], \\
& 2 c g_{01}=\left(1+2 \frac{l_{\alpha}}{r}+2 \frac{l_{\beta}}{s}\right) 2 \Omega \eta,
\end{aligned}
$$




$$
\begin{gathered}
2 c g_{02}=-\left(1+2 \frac{l_{\alpha}}{r}+2 \frac{l_{\beta}}{s}\right) 2 \Omega \xi-8 \frac{\Omega^{2} R}{(1+\rho)}\left(\rho \frac{l_{\alpha}}{r}-\frac{l_{\beta}}{s}\right), \\
g_{03}=0, \\
g_{i j}=-\left(1+2 \frac{l_{\alpha}}{r}+2 \frac{l_{\beta}}{s}\right) \delta_{i j}, \quad i, j=1,2,3 .
\end{gathered}
$$

$l_{\alpha}$ and $l_{\beta}$ being the gravitational radii of the Earth and the Moon, respectively, $r$ and $s$ their respective distances from the planetoid, $\rho$ the ratio between the masses of the Earth and the Moon, $R$ their distance, and

$$
\Omega \equiv \omega\left[1-\frac{3}{2} \frac{\left(l_{\alpha}+l_{\beta}\right)}{R}\left(1-\frac{1}{3} \frac{\rho}{(1+\rho)^{2}}\right)\right],
$$

where $\omega$ represents the Newtonian pulsation.

However, since effective field theories of gravity provide quantum corrections to the Newtonian potential among bodies but not to its powers, we now consider a more refined prescription where only the purely Newtonian terms (i.e., those which are linear or bilinear in $U_{\alpha}$ and $U_{\beta}$ ) are corrected through the map (5.5) and (5.6), while the remaining ones are left unchanged. According to this new perspective, the effective gravity Lagrangian $L_{V}$ can be obtained from (5.7)-(5.12) by setting

$$
\begin{aligned}
& U_{\alpha} \rightarrow V_{\alpha}, \\
& U_{\beta} \rightarrow V_{\beta}, \\
& U_{\alpha} U_{\beta} \rightarrow V_{\alpha} V_{\beta}, \\
& \left(U_{\alpha}\right)^{n} \rightarrow\left(U_{\alpha}\right)^{n}, \quad n>1, \\
& \left(U_{\beta}\right)^{n} \rightarrow\left(U_{\beta}\right)^{n}, \quad n>1 .
\end{aligned}
$$

The new map (5.14) is such that the quantum corrected Lagrangian reads as

$$
\begin{aligned}
L_{V} & =\frac{c^{2}}{2}\left\{1-2\left(V_{\alpha}+V_{\beta}\right)-\frac{\Omega^{2}}{c^{2}}\left(\xi^{2}+\eta^{2}\right)+2\left[\left(U_{\alpha}\right)^{2}+\left(U_{\beta}\right)^{2}\right]\right. \\
& -2 \frac{\left(l_{\alpha}+l_{\beta}\right)}{R^{3}}\left(\xi^{2}+\eta^{2}\right)\left(V_{\alpha}+V_{\beta}\right)+4 V_{\alpha} V_{\beta} \\
& +\frac{(2-\rho)}{(1+\rho)} \frac{l_{\beta}}{R} V_{\alpha}+\frac{(2 \rho-1)}{(1+\rho)} \frac{l_{\alpha}}{R} V_{\beta}-7 \frac{\xi}{R^{2}}\left(l_{\beta} V_{\alpha}-l_{\alpha} V_{\beta}\right) \\
& \left.+(1+\rho)^{-1} \frac{\eta^{2}}{R}\left[\rho \frac{l_{\beta}}{\left(l_{\alpha}\right)^{2}}\left(U_{\alpha}\right)^{3}+\frac{l_{\alpha}}{\left(l_{\beta}\right)^{2}}\left(U_{\beta}\right)^{3}\right]\right\} \\
& -\frac{1}{2}\left(\dot{\xi}^{2}+\dot{\eta}^{2}+\dot{\zeta}^{2}\right)\left[1+2\left(V_{\alpha}+V_{\beta}\right)\right]+\Omega \eta \dot{\xi}\left[1+2\left(V_{\alpha}+V_{\beta}\right)\right] \\
& -\Omega \xi \dot{\eta}\left[1+2\left(V_{\alpha}+V_{\beta}\right)\right]-4 \frac{\Omega^{2} R}{(1+\rho)} \dot{\eta}\left(\rho V_{\alpha}-V_{\beta}\right) .
\end{aligned}
$$

Therefore, new values of quantum corrections on the relativistic distances in the Earth-Moon system [4]

$$
\begin{aligned}
& r_{1, G R}=3.2637628817407598555 \times 10^{8} \mathrm{~m}, \\
& r_{2, G R}=4.4892056003414800050 \times 10^{8} \mathrm{~m}, \\
& r_{3, G R}=3.8167471569392170594 \times 10^{8} \mathrm{~m}, \\
& r_{4, G R}=r_{5, G R}=3.8439999999998611069 \times 10^{8} \mathrm{~m},
\end{aligned}
$$

are found and displayed in Tab. II. Needless to say, the theoretical expression and value of such quantum corrections (if they exist) to Lagrangian points remain an open problem, because the classical effacement property holds only approximately [20,28,31], while 3 sets of quantum parameters $\kappa_{1}$ and $\kappa_{2}$ are conceivable in effective gravity [5, $6,11-13]$. In this respect, the quantum corrections to general relativity values for noncollinear points $L_{4}$ and $L_{5}$ look encouraging in the case of $\left(\kappa_{1}, \kappa_{2}\right)$ values appropriate to scattering potential, because corrections just below a centimeter are comparable with the purely instrumental, time-of-flight uncertainty of the geodesic positioning techniques based on laser-ranging [78-90]. However, the total error budget of satellite/lunar laser ranging [78-90] varies with the specific application and/or orbit, at the level of millimeter to centimeter. 
TABLE II: Quantum corrections on the relativistic position of Earth-Moon Lagrangian points for three different types of potential obtained by considering the Lagrangian function (5.15).

\begin{tabular}{|c|c|c|c|}
\hline \multicolumn{4}{|c|}{ Quantum corrections on Lagrangian points } \\
\hline$L_{i}$ & One-particle reducible & Scattering & Bound-states \\
\hline$L_{1}$ & $r_{Q}-r_{G R}=-1.27 \mathrm{~mm}$ & $r_{Q}-r_{G R}=3.67 \mathrm{~mm}$ & $r_{Q}-r_{G R}=-0.65 \mathrm{~mm}$ \\
\hline$L_{2}$ & $r_{Q}-r_{G R}=-0.75 \mathrm{~mm}$ & $r_{Q}-r_{G R}=2.39 \mathrm{~mm}$ & $r_{Q}-r_{G R}=-0.35 \mathrm{~mm}$ \\
\hline$L_{3}$ & $r_{Q}-r_{G R}=-2.96 \mathrm{~mm}$ & $r_{Q}-r_{G R}=8.89 \mathrm{~mm}$ & $r_{Q}-r_{G R}=-1.48 \mathrm{~mm}$ \\
\hline$L_{4}$ & $r_{Q}-r_{G R}=-2.98 \mathrm{~mm}$ & $r_{Q}-r_{G R}=8.85 \mathrm{~mm}$ & $r_{Q}-r_{G R}=-1.50 \mathrm{~mm}$ \\
\hline$L_{5}$ & $r_{Q}-r_{G R}=-2.98 \mathrm{~mm}$ & $r_{Q}-r_{G R}=8.85 \mathrm{~mm}$ & $r_{Q}-r_{G R}=-1.50 \mathrm{~mm}$ \\
\hline
\end{tabular}

\section{CONCLUDING REMARKS AND OPEN PROBLEMS}

In the first part of our paper, to prepare the ground for future work, we have provided an original synthesis of the Levi-Civita analysis of the problem of motion of $N$ bodies in general relativity, a very difficult problem that was studied, among the others, by Lorenz and Droste [17], Einstein himself with Infeld and Hoffman [18], Fock [19], Levi-Civita [20], Damour, Soffel and Xu [33-36], Kopeikin and Vlasov [49].

The Sun-Earth, Sun-Earth-Moon and $N$-body dynamics have been investigated in Sec. III, while Sec. IV contains our original proof that the nonlinear ordinary differential equations for planetary motions can be always mapped into an exact, linear system of ordinary differential equations, where the unknowns are the fourth-order time derivatives of the original unknown functions. In Sec. V, the evaluation of quantum corrections to location of collinear and noncollinear Lagrangian points for the planar restricted three-body problem has been revisited, and a new set of theoretical values of such corrections for the Earth-Moon-planetoid system has been displayed. It is clear from Tab. II that the few millimeters quantum corrections regarding the relativistic position of Lagrangian points represent a huge obstacle for future experimental measurements. Nevertheless, Einstein theory produces more pronounced (classical) effects on larger distances than those involved in the Earth-Moon system, but at the cost of increasing the efforts for reaching more distant planets. As an example, by applying the framework based on the Lagrangian function (5.7)-(5.12) (see Ref. [4] for further details) to the Sun-Earth and Sun-Jupiter systems we obtain the corrections reported in Tabs. III and IV [94].

Notably, the values of Tab. III agree with those of Ref. [35], and those of Tab. IV with the ones reported in Refs. $[56,60]$. Moreover, we are aware of the fact that many satellites are currently situated near the Sun, but unluckily none of them is planned to approach the Lagrangian point $L_{1}$ in order to test our theoretical model. Eventually, the situation becomes far more complicated for the Sun-Jupiter system because of the large distances involved. Since both the classical and quantum corrections to Lagrangian points of the Earth-Moon system are too small to test general relativity by current observations, it will be important to investigate strong-field effects, e.g. such as the ones in black-hole binaries, where higher order corrections and gravitational radiation must be taken into account. In such cases, however, the weak-field formalism employed in our paper is no longer valid, and one has to use instead the techniques in Refs. [42, 44, 50, 52, 54, 55, 64-67].

In the years to come, we hope that our result in Sec. IV, jointly with the qualitative methods [96] for linear second-order ordinary differential equations in canonical form (see (B4) and (B5)), will lead to improved theoretical calculations of planetary motions in the solar system, with a wide range of applications in fundamental and applied science.

Last but not least, it will be also very interesting to evaluate planetary motions according to the formalism in Refs. [33-36], because it is extremely well suited for studying all departures from the simplifying assumptions made in Ref. [20]. The consideration of all relativistic and tidal moments of all planets, moons and asteroids in the solar system would require a huge computational effort, but meanwhile our qualitative and original results in Sec. IV can be seen as a first step towards solving a relativistic system of equations of motion in the solar system. As a matter of fact, according to the scheme obtained in Refs. [33-36], the exact equations of motion will be integro-differential. 
TABLE III: General relativity corrections on the position of Newtonian Lagrangian points for the Sun-Earth system obtained by considering the general relativity Lagrangian (5.7-5.12). The differences involved refer to the distances of the Sun from the planetoid.

\begin{tabular}{|c|c|}
\hline \multicolumn{2}{|c|}{ General relativity corrections on the Sun-Earth system } \\
\hline$L_{i}$ & Corrections \\
\hline$L_{1}$ & $r_{G R}-r_{c l}=4.8 \mathrm{~m}$ \\
\hline$L_{2}$ & $r_{G R}-r_{c l}=-5.0 \mathrm{~m}$ \\
\hline$L_{3}$ & $r_{G R}-r_{c l}=-0.3 \mathrm{~cm}$ \\
\hline$L_{4,5}$ & $r_{G R}-r_{c l}=-0.3 \mathrm{~cm}$ \\
\hline
\end{tabular}

TABLE IV: General relativity corrections on the position of Newtonian Lagrangian points for the Sun-Jupiter system obtained by employing the general relativity Lagrangian (5.7-5.12). The differences involved refer to the distances of the Sun from the planetoid.

\begin{tabular}{|c|c|}
\hline \multicolumn{2}{|c|}{ General relativity corrections on the Sun-Jupiter system } \\
\hline$L_{i}$ & Corrections \\
\hline$L_{1}$ & $r_{G R}-r_{c l}=30 \mathrm{~m}$ \\
\hline$L_{2}$ & $r_{G R}-r_{c l}=-38 \mathrm{~m}$ \\
\hline$L_{3}$ & $r_{G R}-r_{c l}=-1 \mathrm{~m}$ \\
\hline$L_{4,5}$ & $r_{G R}-r_{c l}=-1 \mathrm{~m}$ \\
\hline
\end{tabular}

\section{APPENDIX A: LINEAR DIFFERENTIAL EQUATIONS ASSOCIATED TO NONLINEAR ONES}

In this appendix we provide the details of the calculations leading to (4.8) starting from Eq (3.31) written in the form given by (4.3). As explained in Sec. IV, bearing in mind Eqs. (4.4) and (4.5), the first- and second-order time derivatives of (4.3) are given by Eqs. (4.6) and (4.7), respectively.

The third time derivative of (4.3) gives a nonlinear system of differential equations with unknowns $x_{p}^{(3)}(p=1,2)$ 
having the form

$$
\begin{aligned}
& \mathcal{A}_{i r} \frac{\mathrm{d}^{2}}{\mathrm{~d} t^{2}} x_{r}^{(3)}+3\left(\frac{\partial \mathcal{A}_{i r}}{\partial x_{j}} \dot{x}_{j}+\frac{\partial \mathcal{A}_{i r}}{\partial \dot{x}_{j}} \ddot{x}_{j}\right) \frac{\mathrm{d}}{\mathrm{d} t} x_{r}^{(3)}+\ddot{x}_{r} \frac{\partial \mathcal{A}_{i r}}{\partial \dot{x}_{k}} \frac{\mathrm{d}}{\mathrm{d} t} x_{k}^{(3)}+\frac{\partial \mathcal{H}_{i}}{\partial \dot{x}_{k}} \frac{\mathrm{d}}{\mathrm{d} t} x_{k}^{(3)}+2\left[\left(\frac{\mathrm{d}}{\mathrm{d} t} \frac{\partial \mathcal{A}_{i r}}{\partial x_{j}}\right) \dot{x}_{j}+\frac{\partial \mathcal{A}_{i r}}{\partial x_{j}} \ddot{x}_{j}\right. \\
& \left.+\left(\frac{\mathrm{d}}{\mathrm{d} t} \frac{\partial \mathcal{A}_{i r}}{\partial \dot{x}_{j}}\right) \ddot{x}_{j}+\left(\frac{\partial \mathcal{A}_{i r}}{\partial \dot{x}_{j}}\right) x_{j}^{(3)}\right] x_{r}^{(3)}+x_{r}^{(3)} \frac{\partial \mathcal{A}_{i r}}{\partial \dot{x}_{k}} x_{k}^{(3)}+\ddot{x}_{r}\left(\frac{\mathrm{d}}{\mathrm{d} t} \frac{\partial \mathcal{A}_{i r}}{\partial \dot{x}_{k}}\right) x_{k}^{(3)}+\left(\frac{\mathrm{d}}{\mathrm{d} t} \frac{\partial \mathcal{H}_{i}}{\partial \dot{x}_{k}}\right) x_{k}^{(3)}+\left[\left(\frac{\mathrm{d}}{\mathrm{d} t} \frac{\partial^{2} \mathcal{A}_{i r}}{\partial x_{l} \partial x_{k}}\right) \dot{x}_{l} \dot{x}_{k}\right. \\
& +\left(\frac{\partial^{2} \mathcal{A}_{i r}}{\partial x_{l} \partial x_{k}}\right)\left(\ddot{x}_{l} \dot{x}_{k}+\dot{x}_{l} \ddot{x}_{k}\right)+2\left(\frac{\mathrm{d}}{\mathrm{d} t} \frac{\partial^{2} \mathcal{A}_{i r}}{\partial \dot{x}_{l} \partial x_{k}}\right) \ddot{x}_{l} \dot{x}_{k}+2\left(\frac{\partial^{2} \mathcal{A}_{i r}}{\partial \dot{x}_{l} \partial x_{k}}\right)\left(x_{l}^{(3)} \dot{x}_{k}+\ddot{x}_{l} \ddot{x}_{k}\right)+\left(\frac{\mathrm{d}}{\mathrm{d} t} \frac{\partial \mathcal{A}_{i r}}{\partial x_{k}}\right) \ddot{x}_{k} \\
& \left.+\left(\frac{\partial \mathcal{A}_{i r}}{\partial x_{k}}\right) x_{k}^{(3)}+\left(\frac{\mathrm{d}}{\mathrm{d} t} \frac{\partial^{2} \mathcal{A}_{i r}}{\partial \dot{x}_{k} \partial \dot{x}_{l}}\right) \ddot{x}_{l} \ddot{x}_{k}+\left(\frac{\partial^{2} \mathcal{A}_{i r}}{\partial \dot{x}_{k} \partial \dot{x}_{l}}\right)\left(x_{l}^{(3)} \ddot{x}_{k}+\ddot{x}_{l} x_{k}^{(3)}\right)\right] \ddot{x}_{r}+\left(\frac{\partial^{2} \mathcal{A}_{i r}}{\partial x_{l} \partial x_{k}} \dot{x}_{l} \dot{x}_{k}+2 \frac{\partial^{2} \mathcal{A}_{i r}}{\partial \dot{x}_{l} \partial x_{k}} \ddot{x}_{l} \dot{x}_{k}\right. \\
& \left.+\frac{\partial \mathcal{A}_{i r}}{\partial x_{k}} \ddot{x}_{k}+\frac{\partial^{2} \mathcal{A}_{i r}}{\partial \dot{x}_{l} \partial \dot{x}_{k}} \ddot{x}_{l} \ddot{x}_{k}\right) x_{r}^{(3)}+\left(\frac{\mathrm{d}}{\mathrm{d} t} \frac{\partial^{2} \mathcal{H}_{i}}{\partial x_{l} \partial x_{k}}\right) \dot{x}_{l} \dot{x}_{k}+\frac{\partial^{2} \mathcal{H}_{i}}{\partial x_{l} \partial x_{k}}\left(\ddot{x}_{l} \dot{x}_{k}+\dot{x}_{l} \ddot{x}_{k}\right)+2\left(\frac{\mathrm{d}}{\mathrm{d} t} \frac{\partial^{2} \mathcal{H}_{i}}{\partial \dot{x}_{l} \partial x_{k}}\right) \ddot{x}_{l} \dot{x}_{k} \\
& +2\left(\frac{\partial^{2} \mathcal{H}_{i}}{\partial \dot{x}_{l} \partial x_{k}}\right)\left(x_{l}^{(3)} \dot{x}_{k}+\ddot{x}_{l} \ddot{x}_{k}\right)+\left(\frac{\mathrm{d}}{\mathrm{d} t} \frac{\partial \mathcal{H}_{i}}{\partial x_{k}}\right) \ddot{x}_{k}+\frac{\partial \mathcal{H}_{i}}{\partial x_{k}} x_{k}^{(3)}+\left(\frac{\mathrm{d}}{\mathrm{d} t} \frac{\partial^{2} \mathcal{H}_{i}}{\partial \dot{x}_{l} \partial \dot{x}_{k}}\right) \ddot{x}_{k} \ddot{x}_{l}+\left(\frac{\partial^{2} \mathcal{H}_{i}}{\partial \dot{x}_{l} \partial \dot{x}_{k}}\right)\left(x_{l}^{(3)} \ddot{x}_{k}+\ddot{x}_{l} x_{k}^{(3)}\right)=0, \\
& (i=1,2) \text {. }
\end{aligned}
$$

The nonlinearities occurring in (A1) vanish if we compute the fourth time derivative. In fact, as anticipated in Sec. IV, by differentiating once again Eq. (A1) we end up with a linear system of coupled ordinary differential equations for the unknown functions $x_{p}^{(4)}(p=1,2)$ which can be written as

$$
\begin{aligned}
& \mathcal{A}_{i r} \frac{\mathrm{d}^{2}}{\mathrm{~d} t^{2}} x_{r}^{(4)}+4\left(\frac{\partial \mathcal{A}_{i r}}{\partial x_{j}} \dot{x}_{j}+\frac{\partial \mathcal{A}_{i r}}{\partial \dot{x}_{j}} \ddot{x}_{j}\right) \frac{\mathrm{d}}{\mathrm{d} t} x_{r}^{(4)}+\ddot{x}_{r}\left(\frac{\partial \mathcal{A}_{i r}}{\partial \dot{x}_{k}}\right) \frac{\mathrm{d}}{\mathrm{d} t} x_{k}^{(4)}+\left(\frac{\partial \mathcal{H}_{i}}{\partial \dot{x}_{k}}\right) \frac{\mathrm{d}}{\mathrm{d} t} x_{k}^{(4)}+F_{i}\left(x_{p}, \dot{x}_{p}, \ldots, x_{p}^{(4)}\right)=0, \\
& (i, p=1,2) .
\end{aligned}
$$


where

$$
\begin{aligned}
& F_{i}\left(x_{p}, \dot{x}_{p}, \ldots, x_{p}^{(4)}\right)=3\left[\frac{\mathrm{d}}{\mathrm{d} t}\left(\frac{\partial \mathcal{A}_{i r}}{\partial x_{j}} \dot{x}_{j}+\frac{\partial \mathcal{A}_{i r}}{\partial \dot{x}_{j}} \ddot{x}_{j}\right)\right] x_{r}^{(4)}+2\left\{\frac { \mathrm { d } } { \mathrm { d } t } \left[\left(\frac{\mathrm{d}}{\mathrm{d} t} \frac{\partial \mathcal{A}_{i r}}{\partial x_{j}}\right) \dot{x}_{j}+\frac{\partial \mathcal{A}_{i r}}{\partial x_{j}} \ddot{x}_{j}+\left(\frac{\mathrm{d}}{\mathrm{d} t} \frac{\partial \mathcal{A}_{i r}}{\partial \dot{x}_{j}}\right) \ddot{x}_{j}\right.\right. \\
& \left.\left.+\left(\frac{\partial \mathcal{A}_{i r}}{\partial \dot{x}_{j}}\right) x_{j}^{(3)}\right]\right\} x_{r}^{(3)}+2\left[\left(\frac{\mathrm{d}}{\mathrm{d} t} \frac{\partial \mathcal{A}_{i r}}{\partial x_{j}}\right) \dot{x}_{j}+\frac{\partial \mathcal{A}_{i r}}{\partial x_{j}} \ddot{x}_{j}+\left(\frac{\mathrm{d}}{\mathrm{d} t} \frac{\partial \mathcal{A}_{i r}}{\partial \dot{x}_{j}}\right) \ddot{x}_{j}+\left(\frac{\partial \mathcal{A}_{i r}}{\partial \dot{x}_{j}}\right) x_{j}^{(3)}\right] x_{r}^{(4)} \\
& +\left(\frac{\mathrm{d}^{2}}{\mathrm{~d} t^{2}} \frac{\partial \mathcal{A}_{i r}}{\partial \dot{x}_{k}}\right) \ddot{x}_{r} x_{k}^{(3)}+2\left(\frac{\mathrm{d}}{\mathrm{d} t} \frac{\partial \mathcal{A}_{i r}}{\partial \dot{x}_{k}}\right)\left(x_{r}^{(3)} x_{k}^{(3)}+\ddot{x}_{r} x_{k}^{(4)}\right)+\left(\frac{\partial \mathcal{A}_{i r}}{\partial \dot{x}_{k}}\right)\left(x_{r}^{(4)} x_{k}^{(3)}+2 x_{r}^{(3)} x_{k}^{(4)}\right) \\
& +\left(\frac{\mathrm{d}^{2}}{\mathrm{~d} t^{2}} \frac{\partial \mathcal{H}_{i}}{\partial \dot{x}_{k}}\right) x_{k}^{(3)}+2\left(\frac{\mathrm{d}}{\mathrm{d} t} \frac{\partial \mathcal{H}_{i}}{\partial \dot{x}_{k}}\right) x_{k}^{(4)}+\left\{\frac { \mathrm { d } } { \mathrm { d } t } \left[\left(\frac{\mathrm{d}}{\mathrm{d} t} \frac{\partial^{2} \mathcal{A}_{i r}}{\partial x_{l} \partial x_{k}}\right) \dot{x}_{l} \dot{x}_{k}+\left(\frac{\partial^{2} \mathcal{A}_{i r}}{\partial x_{l} \partial x_{k}}\right)\left(\ddot{x}_{l} \dot{x}_{k}+\dot{x}_{l} \ddot{x}_{k}\right)\right.\right. \\
& +2\left(\frac{\mathrm{d}}{\mathrm{d} t} \frac{\partial^{2} \mathcal{A}_{i r}}{\partial \dot{x}_{l} \partial x_{k}}\right) \ddot{x}_{l} \dot{x}_{k}+2\left(\frac{\partial^{2} \mathcal{A}_{i r}}{\partial \dot{x}_{l} \partial x_{k}}\right)\left(x_{l}^{(3)} \dot{x}_{k}+\ddot{x}_{l} \ddot{x}_{k}\right)+\left(\frac{\mathrm{d}}{\mathrm{d} t} \frac{\partial \mathcal{A}_{i r}}{\partial x_{k}}\right) \ddot{x}_{k}+\left(\frac{\partial \mathcal{A}_{i r}}{\partial x_{k}}\right) x_{k}^{(3)} \\
& \left.\left.+\left(\frac{\mathrm{d}}{\mathrm{d} t} \frac{\partial^{2} \mathcal{A}_{i r}}{\partial \dot{x}_{k} \partial \dot{x}_{l}}\right) \ddot{x}_{l} \ddot{x}_{k}+\left(\frac{\partial^{2} \mathcal{A}_{i r}}{\partial \dot{x}_{k} \partial \dot{x}_{l}}\right)\left(x_{l}^{(3)} \ddot{x}_{k}+\ddot{x}_{l} x_{k}^{(3)}\right)\right]\right\} \ddot{x}_{r}+\left[\left(\frac{\mathrm{d}}{\mathrm{d} t} \frac{\partial^{2} \mathcal{A}_{i r}}{\partial x_{l} \partial x_{k}}\right) \dot{x}_{l} \dot{x}_{k}\right. \\
& +\left(\frac{\partial^{2} \mathcal{A}_{i r}}{\partial x_{l} \partial x_{k}}\right)\left(\ddot{x}_{l} \dot{x}_{k}+\dot{x}_{l} \ddot{x}_{k}\right)+2\left(\frac{\mathrm{d}}{\mathrm{d} t} \frac{\partial^{2} \mathcal{A}_{i r}}{\partial \dot{x}_{l} \partial x_{k}}\right) \ddot{x}_{l} \dot{x}_{k}+2\left(\frac{\partial^{2} \mathcal{A}_{i r}}{\partial \dot{x}_{l} \partial x_{k}}\right)\left(x_{l}^{(3)} \dot{x}_{k}+\ddot{x}_{l} \ddot{x}_{k}\right) \\
& \left.+\left(\frac{\mathrm{d}}{\mathrm{d} t} \frac{\partial \mathcal{A}_{i r}}{\partial x_{k}}\right) \ddot{x}_{k}+\left(\frac{\partial \mathcal{A}_{i r}}{\partial x_{k}}\right) x_{k}^{(3)}+\left(\frac{\mathrm{d}}{\mathrm{d} t} \frac{\partial^{2} \mathcal{A}_{i r}}{\partial \dot{x}_{k} \partial \dot{x}_{l}}\right) \ddot{x}_{l} \ddot{x}_{k}+\left(\frac{\partial^{2} \mathcal{A}_{i r}}{\partial \dot{x}_{k} \partial \dot{x}_{l}}\right)\left(x_{l}^{(3)} \ddot{x}_{k}+\ddot{x}_{l} x_{k}^{(3)}\right)\right] x_{r}^{(3)} \\
& +\left[\frac{\mathrm{d}}{\mathrm{d} t}\left(\frac{\partial^{2} \mathcal{A}_{i r}}{\partial x_{l} \partial x_{k}} \dot{x}_{l} \dot{x}_{k}+2 \frac{\partial^{2} \mathcal{A}_{i r}}{\partial \dot{x}_{l} \partial x_{k}} \ddot{x}_{l} \dot{x}_{k}+\frac{\partial \mathcal{A}_{i r}}{\partial x_{k}} \ddot{x}_{k}+\frac{\partial^{2} \mathcal{A}_{i r}}{\partial \dot{x}_{l} \partial \dot{x}_{k}} \ddot{x}_{l} \ddot{x}_{k}\right)\right] x_{r}^{(3)}+\left(\frac{\partial^{2} \mathcal{A}_{i r}}{\partial x_{l} \partial x_{k}} \dot{x}_{l} \dot{x}_{k}\right. \\
& \left.+2 \frac{\partial^{2} \mathcal{A}_{i r}}{\partial \dot{x}_{l} \partial x_{k}} \ddot{x}_{l} \dot{x}_{k}+\frac{\partial \mathcal{A}_{i r}}{\partial x_{k}} \ddot{x}_{k}+\frac{\partial^{2} \mathcal{A}_{i r}}{\partial \dot{x}_{l} \partial \dot{x}_{k}} \ddot{x}_{l} \ddot{x}_{k}\right) x_{r}^{(4)}+\left(\frac{\mathrm{d}^{2}}{\mathrm{~d} t^{2}} \frac{\partial^{2} \mathcal{H}_{i}}{\partial x_{l} \partial x_{k}}\right) \dot{x}_{l} \dot{x}_{k} \\
& +2\left(\frac{\mathrm{d}}{\mathrm{d} t} \frac{\partial^{2} \mathcal{H}_{i}}{\partial x_{l} \partial x_{k}}\right)\left(\ddot{x}_{l} \dot{x}_{k}+\dot{x}_{l} \ddot{x}_{k}\right)+\left(\frac{\partial^{2} \mathcal{H}_{i}}{\partial x_{l} \partial x_{k}}\right)\left(x_{l}^{(3)} \dot{x}_{k}+2 \ddot{x}_{l} \ddot{x}_{k}+\dot{x}_{l} x_{k}^{(3)}\right)+2\left(\frac{\mathrm{d}^{2}}{\mathrm{~d} t^{2}} \frac{\partial^{2} \mathcal{H}_{i}}{\partial \dot{x}_{l} \partial x_{k}}\right) \ddot{x}_{l} \dot{x}_{k} \\
& +4\left(\frac{\mathrm{d}}{\mathrm{d} t} \frac{\partial^{2} \mathcal{H}_{i}}{\partial \dot{x}_{l} \partial x_{k}}\right)\left(x_{l}^{(3)} \dot{x}_{k}+\ddot{x}_{l} \ddot{x}_{k}\right)+2\left(\frac{\partial^{2} \mathcal{H}_{i}}{\partial \dot{x}_{l} \partial x_{k}}\right)\left(x_{l}^{(4)} \dot{x}_{k}+2 x_{l}^{(3)} \ddot{x}_{k}+\ddot{x}_{l} x_{k}^{(3)}\right)+\left(\frac{\mathrm{d}^{2}}{\mathrm{~d} t^{2}} \frac{\partial \mathcal{H}_{i}}{\partial x_{k}}\right) \ddot{x}_{k} \\
& +2\left(\frac{\mathrm{d}}{\mathrm{d} t} \frac{\partial \mathcal{H}_{i}}{\partial x_{k}}\right) x_{k}^{(3)}+\frac{\partial \mathcal{H}_{i}}{\partial x_{k}} x_{k}^{(4)}+\left(\frac{\mathrm{d}^{2}}{\mathrm{~d} t^{2}} \frac{\partial^{2} \mathcal{H}_{i}}{\partial \dot{x}_{l} \partial \dot{x}_{k}}\right) \ddot{x}_{l} \ddot{x}_{k}+2\left(\frac{\mathrm{d}}{\mathrm{d} t} \frac{\partial^{2} \mathcal{H}_{i}}{\partial \dot{x}_{l} \partial \dot{x}_{k}}\right)\left(x_{l}^{(3)} \ddot{x}_{k}+\ddot{x}_{l} x_{k}^{(3)}\right) \\
& +\frac{\partial^{2} \mathcal{H}_{i}}{\partial \dot{x}_{l} \partial \dot{x}_{k}}\left(x_{l}^{(4)} \ddot{x}_{k}+2 x_{k}^{(3)} x_{l}^{(3)}+\ddot{x}_{l} x_{k}^{(4)}\right), \quad(i, p=1,2) .
\end{aligned}
$$

It is thus clear from last equation that the functions $F_{i}\left(x_{p}, \dot{x}_{p}, \ldots, x_{p}^{(4)}\right)(i=1,2)$ depend linearly on $x_{p}^{(4)}(p=1,2)$ and that no derivatives of order higher than four appear, which clearly means that Eq. (A2) (or equivalently (4.8)) is linear with respect to $x_{p}^{(4)} .^{8}$

${ }^{8}$ Recall that the most general form of a linear ordinary differential equation of order $n$ is given by

$$
\left(r_{n}(t) \frac{\mathrm{d}^{n}}{\mathrm{~d} t^{n}}+r_{n-1}(t) \frac{\mathrm{d}^{n-1}}{\mathrm{~d} t^{n-1}}+\cdots+r_{1}(t) \frac{\mathrm{d}}{\mathrm{d} t}+r_{0}(t)\right) x(t)=f(t)
$$

the coefficients $r_{n}(t), r_{n-1}(t), \ldots, r_{1}(t), r_{0}(t)$ and the term $f(t)$ being continuous real-valued functions of $t$ in the interval $a \leq t \leq b$ and $r_{n}(t)$ a function that does not vanish at any point of the aforementioned interval. The operator

$$
L \equiv r_{n}(t) \frac{\mathrm{d}^{n}}{\mathrm{~d} t^{n}}+r_{n-1}(t) \frac{\mathrm{d}^{n-1}}{\mathrm{~d} t^{n-1}}+\cdots+r_{1}(t) \frac{\mathrm{d}}{\mathrm{d} t}+r_{0}(t)
$$

is called linear differential operator of order $n$. 


\section{APPENDIX B: LINEAR DIFFERENTIAL EQUATIONS OF SECOND ORDER. STURM-LIOUVILLE}

PROBLEM

Within the framework of ordinary differential equations, every second-order linear differential equation can be written in the form

$$
\left[\frac{d^{2}}{d x^{2}}+p(x) \frac{d}{d x}+q(x)\right] u(x)=0,
$$

where $x$ is taken to lie in the closed interval $[a, b]$, while $p$ and $q$ are suitably smooth functions. This equation can be brought into the Liouville form, where the coefficient of the first-order derivative vanishes. For this purpose, one sets $u(x)=\varphi(x) \psi(x)$, so that Eq. (B1) reads as

$$
\varphi \psi^{\prime \prime}+\left(2 \varphi^{\prime}+p \varphi\right) \psi^{\prime}+\left(\varphi^{\prime \prime}+p \varphi^{\prime}+q \varphi\right) \psi=0 .
$$

Our task is achieved if the function $\varphi$ solves the first-order equation

$$
\frac{\varphi^{\prime}}{\varphi}=-\frac{p}{2} \Longrightarrow \varphi(x)=\exp \left(-\frac{1}{2} \int p(x) d x\right) \text {. }
$$

At this stage, since we can divide by $\varphi$, we can re-express Eq. (B2) in the form

$$
\psi^{\prime \prime}+W \psi=0,
$$

where the potential term turns out to be

$$
W=\frac{\varphi^{\prime \prime}}{\varphi}+p \frac{\varphi^{\prime}}{\varphi}+q=-\frac{1}{2} p^{\prime}-\frac{p^{2}}{4}+q .
$$

Once we have reduced ourselves to studying Eq. (B4), one can deduce important qualitative properties. For example, if the function $W$ is continuous for $x \in[a, b]$, and if there exist real constants $\omega$ and $\Omega$ such that

$$
0<\omega^{2}<W(x)<\Omega^{2}
$$

one can compare the zeros of solutions of Eq. (B4) with the zeros of solutions of the equations

$$
\psi^{\prime \prime}+\alpha^{2} \psi=0, \quad \alpha=\omega \text { or } \Omega .
$$

Equations (B7) are solved by periodic functions $\sin \alpha\left(x-x_{0}\right)$ which have zeros at $x_{0}+\frac{k \pi}{\alpha}, k$ being an integer and $\alpha$ taking one of the two values in (B7). One can then prove that the difference $\delta$ between two adjacent zeros of a solution of Eq. (B4) satifies the conditions [97]

$$
\frac{\pi}{\Omega} \leq \delta \leq \frac{\pi}{\omega}
$$

Now the first of our Eqs. (3.15) (or the more general (3.31)) can be written, after using the procedure used in Sec. IV, i.e. four differentiations with respect to $t$ of the original equation, in the form

$$
g(t)\left[Y^{\prime \prime}+p_{1}(t) Y^{\prime}+q_{1} Y\right]+f(t)\left[X^{\prime \prime}+p_{2}(t) X^{\prime}+q_{2} X\right]=0,
$$

where, for each pair $\left(p_{1}, q_{1}\right)$ and $\left(p_{2}, q_{2}\right)$, we can evaluate the corresponding potential $W_{1}$ (respectively $\left.W_{2}\right)$ according to Eq. (B5). The letters $X$ and $Y$ here denote fourth-order time derivative of the original functions $x(t)$ and $y(t)$, respectively.

\section{Acknowledgments}

E. B. and G. E. are grateful to the Dipartimento di Fisica "Ettore Pancini" of Federico II University for hospitality and support. The work of E. B., S. D. and G. E. has been supported by the INFN funding of the NEWREFLECTIONS experiment.

[1] E. Battista and G. Esposito, Restricted three-body problem in effective-field-theory models of gravity, Phys. Rev. D 89, 084030 (2014). 
[2] E. Battista and G. Esposito, Full three-body problem in effective-field-theory models of gravity, Phys. Rev. D 90, 084010 (2014); Phys. Rev. D 93, 049901(E) (2016).

[3] E. Battista, S. Dell'Agnello, G. Esposito and J. Simo, Quantum effects on Lagrangian points and displaced periodic orbits in the Earth-Moon system, Phys. Rev. D 91, 084041 (2015); Phys. Rev. D 93, 049902(E) (2016).

[4] E. Battista, S. Dell'Agnello, G. Esposito, L. Di Fiore, J. Simo and A. Grado, Earth-Moon Lagrangian points as a test bed for general relativity and effective field theories of gravity, Phys. Rev. D 92, 064045 (2015); Phys. Rev. D 93, 109904(E) (2016).

[5] J. F. Donoghue, Leading quantum correction to the Newtonian potential, Phys. Rev. Lett. 72, 2996 (1994).

[6] J. F. Donoghue, General relativity as an effective field theory: the leading quantum corrections, Phys. Rev. D 50, 3874 (1994).

[7] I. J. Muzinich and S. Vokos, Long range forces in quantum gravity, Phys. Rev. D 52, 3472 (1995).

[8] H. W. Hamber and S. Liu, On the quantum corrections to the Newtonian potential, Phys. Lett. B 357, 51 (1995).

[9] A. A. Akhundov, S. Bellucci, and A. Shiekh, Gravitational interaction to one loop in effective quantum gravity, Phys. Lett. B 395, 16 (1997).

[10] I. B. Khriplovich and G. G. Kirilin, Quantum power correction to the Newton law, Sov. Phys. JETP 95, 981 (2002).

[11] N. E. J. Bjerrum-Bohr, J. F. Donoghue, and B. R. Holstein, Quantum gravitational corrections to the nonrelativistic scattering potential of two masses, Phys. Rev. D 67, 084033 (2003).

[12] N. E. J. Bjerrum-Bohr, J. F. Donoghue, and B. R. Holstein, Quantum corrections to the Schwarzschild and Kerr metrics, Phys. Rev. D 68, 084005 (2003).

[13] J. F. Donoghue, The effective field theory treatment of quantum gravity, AIP Conf. Proc. 1483, 73 (2012).

[14] F. F. Tisserand, Treatise of Celestial Mechanics, Vols. 1 to 4 (Gauthier-Villars, Paris, 1889-1896; Jacques Gabay, Paris, 1990).

[15] H. Poincaré, The three-body problem and the equations of dynamics, Acta Mathematica 13, 1 (1890); On the three-body problem, Bull. Astronomique 8, 12 (1891).

[16] H. Poincaré, Les Methodes Nouvelles de la Mecanique Celeste (Gauthier-Villars, Paris, 1892), reprinted as New Methods of Celestial Mechanics, edited by D. L. Goroff (American Institute of Physics, College Park, 1993).

[17] H. A. Lorentz and J. Droste, in H. A. Lorentz, Collected papers, edited by P. Zeeman and A. D. Fokker (Nijhoff, The Hague, 1937), Vol. V, pp. 330-355.

[18] A. Einstein, L. Infeld, and B. Hoffman, The gravitational equations and the problem of motion, Ann. Math. 39, 65 (1938).

[19] V. A. Fock, On the motion of finite masses after the Einstein theory of gravitation, J. Phys. (Moscow) 1, 81 (1939).

[20] T. Levi-Civita, The N-Body Problem in General Relativity (Gauthier-Villars, Paris, 1941; Reidel, Dordrecht, 1964).

[21] B. D. Tapley and J. M. Lewallen, Solar influence on satellite motion near the stable earth-moon libration points, AIAA J. 2, 728 (1964).

[22] L. A. Pars, A Treatise on Analytical Dynamics (Heinemann, London, 1965).

[23] V. Szebehely, Theory of Orbits: the Restricted Problem of Three Bodies (Academic Press, New York, 1967).

[24] E. Krefetz, Restricted three-body problem in the post-Newtonian approximation, Astron. J. 72, 471 (1967).

[25] V. A. Brumberg, Relativistic Celestial Mechanics (Nauka, Moscow, 1972).

[26] R. A. Freitas and F. Valdes, A search for natural or artificial objects located at the Earth-Moon libration points, Icarus 42, 442 (1980).

[27] L. Blanchet and T. Damour, Radiative gravitational fields in general relativity I. general structure of the field outside the source, Phil. Trans. Roy. Soc. Lond. A 320, 379 (1986).

[28] T. Damour, The problem of motion in Newtonian and Einsteinian gravity, in 300 Years of Gravitation, eds. S. W. Hawking and W. Israel (Cambridge University Press, Cambridge, 1987).

[29] L. Blanchet and T. Damour, Post-Newtonian generation of gravitational waves, Ann. Inst. Henri Poincaré 50, 377 (1989).

[30] T. Damour and B. R. Iyer, Multipole analysis for electromagnetism and linearized gravity with irreducible Cartesian tensors, Phys. Rev. D 43, 3259 (1991).

[31] T. Damour and G. Schafer, Levi-Civita and the general relativistic problem of motion, in Studies in the History of General Relativity, Einstein Studies Vol. 3, eds. J. Eisenstaedt, A. J. Kox (Birkhauser, Boston, 1992).

[32] A. Celletti and A. Giorgilli, On the stability of the Lagrangian points in the spatial restricted problem of three bodies, Cel. Mech. Dyn. Astron. 50, 31 (1991).

[33] T. Damour, M. Soffel, and C. Xu, General-relativistic celestial mechanics. I. Method and definition of reference systems, Phys. Rev. D 43, 3273 (1991).

[34] T. Damour, M. Soffel, and C. Xu, General-relativistic celestial mechanics. II. Translational equations of motion, Phys. Rev. D 45, 1017 (1992).

[35] T. Damour, M. Soffel, and C. Xu, General-relativistic celestial mechanics. III. Rotational equations of motion, Phys. Rev. D 47, 3124 (1993).

[36] T. Damour, M. Soffel, and C. Xu, General-relativistic celestial mechanics. IV. Theory of satellite motion, Phys. Rev. D 49, 618 (1994).

[37] T. I. Maindl and R. Dvorak, On the dynamics of the relativistic restricted three-body problem, Astronomy \& Astrophys. 290, 335 (1994).

[38] T. I. Maindl, The solar system's Lagrangian points in the framework of the relativistic restricted three-body problem, ASP Conference Series 107, 147 (1996).

[39] A. Celletti and L. Chierchia, On the stability of realistic three-body problems, Commun. Math. Phys. 186, 413 (1997).

[40] G. Dell'Antonio, Noncollision periodic solutions of the N-body system, Nonlinear Differ. Equ. Appl. 5, 117 (1998). 
[41] K. B. Bhatnagar and P. P. Hallan, Existence and stability of $L_{4,5}$ in the relativistic restricted three-body problem, Cel. Mech. Dyn. Astron. 69, 271 (1998).

[42] A. Buonanno and T. Damour, Effective one-body approach to general relativistic two-body dynamics, Phys. Rev. D 59, 084006 (1999).

[43] C. D. Murray and S. F. Dermott, Solar System Dynamics (Cambridge University Press, Cambridge, 1999).

[44] A. Buonanno and T. Damour, Transition from inspiral to plunge in binary black hole coalescences, Phys. Rev. D 62, 064015 (2000).

[45] C. N. Douskos and E. A. Perdios, On the stability of equilibrium points in the relativistic restricted three-body problem, Cel. Mech. Dyn. Astron. 82, 317 (2002).

[46] A. Morbidelli, Modern Celestial Mechanics: Aspects of Solar System Dynamics (Taylor \& Francis, London, 2002).

[47] V. A. Brumberg, Special solutions in a simplified restricted three-body problem with gravitational radiation taken into account, Cel. Mech. Dyn. Astron. 85, 269 (2003).

[48] L. F. Wanex, Chaotic amplification in the relativistic restricted three-body problem, Z. Naturforsch. 58a, 13 (2003).

[49] S. Kopeikin and I. Vlasov, Parametrized post-Newtonian theory of reference frames, multipolar expansion and equations of motion in the N-body problem, Phys. Rep. 400, 209 (2004).

[50] A. Buonanno, Y. Chen, and T. Damour, Transition from inspiral to plunge in precessing binaries of spinning black holes, Phys. Rev. D 74, 104005 (2006).

[51] S. Kopeikin and I. Vlasov, The effacing principle in the post-Newtonian celestial mechanics, in Proceedings XI Marcel Grossmann Meeting, 2475-2477 (World Scientific, Singapore, 2008).

[52] T. Damour, A. Nagar, M. Hannam, S. Husa, and B. Bruegmann, Accurate effective-one-body waveforms of inspiralling and coalescing black-hole binaries, Phys. Rev. D 78, 044039 (2008).

[53] H. Asada, Gravitational wave forms for a three-body system in Lagrange's orbit: Parameter determinations and a binary source test, Phys. Rev. D 80, 064021 (2009).

[54] T. Damour and A. Nagar, An improved analytical description of inspiralling and coalescing black-hole binaries, arXiv:0902.0136.

[55] T. Damour, B. R. Iyer, and A. Nagar, Improved resummation of post-Newtonian multipolar waveforms from circularized compact binaries, Phys. Rev. D 79, 064004 (2009).

[56] K. Yamada and H. Asada, Collinear solution to the general relativistic three-body problem, Phys. Rev. D 82, 104019 (2010).

[57] H. Asada, T. Futamase and P. Hogan, Equations of Motion in General Relativity, Int. Ser. Monogr. Phys. 148 (Oxford University Press, Oxford, 2010).

[58] M. Connors, P. Wiegert, and C. Veillet, Earth's Trojan asteroid, Nature (London) 475, 481 (2011).

[59] B. Bertotti, P. Farinella and D. Vokrouhlicky, Physics of the Solar System: Dynamics and Evolution, Space Physics, and Spacetime Structure (Springer, Berlin, 2012).

[60] K. Yamada and H. Asada, Triangular solution to the general relativistic three-body problem for general masses, Phys. Rev. D 86, 124029 (2012).

[61] T. Damour, The general relativistic two-body problem, arXiv:1312.3505 [gr-qc].

[62] List of Jupiter Trojans, Minor Planet Center, 25 February 2014.

[63] K. Yamada and H. Asada, Post-Newtonian effects on the stability of the triangular solution in the three-body problem for general masses, Phys. Rev. D 91, 124016 (2015).

[64] D. Bini, T. Damour, and A. Geralico, Confirming and improving post-Newtonian and effective-one-body results from self-force computations along eccentric orbits around a Schwarzschild black hole, Phys. Rev. D 93, 064023 (2016).

[65] D. Bini, T. Damour, and A. Geralico, New gravitational self-force analytical results for eccentric orbits around a Schwarzschild black hole, Phys. Rev. D 93, 104017 (2016).

[66] T. Damour and D. Bini, Conservative second-order gravitational self-force on circular orbits and the effective one-body formalism, Phys. Rev. D 93, 104040 (2016).

[67] D. Bini, T. Damour, and A. Geralico, High post-Newtonian order gravitational self-force analytical results for eccentric equatorial orbits around a Kerr black hole, Phys. Rev. D 93, 124058 (2016).

[68] K. Yamada and H. Asada, Non-chaotic evolution of triangular configuration due to gravitational radiation reaction in the three-body problem, Phys. Rev. D 93, 084027 (2016).

[69] T. Y. Zhou, W. G. Cao, and Y. Xie, Collinear solution to the three-body problem under a scalar-tensor gravity, Phys. Rev. D 93, 064065 (2016).

[70] C. R. McInnes, Solar Sailing: Technology, Dynamics and Mission Applications (Springer Praxis, London, 1999).

[71] J. Simo and C. R. McInnes, Solar sail trajectories at the earth-moon Lagrange points, in 59th International Astronomical Congress, Glasgow, Scotland, 2008.

[72] J. Simo and C. R. McInnes, Displaced periodic orbits with low-thrust propulsion in the earth-moon system, in 19th AAS/AIAA Space Flight Mechanics Meeting, Savannah, Georgia, 2009.

[73] J. Simo and C. R. McInnes, Solar sail orbits at the earth-moon libration points, Comm. Nonlinear Sci. Numer. Simulat. 14, 4191 (2009).

[74] J. Simo and C. R. McInnes, Asymptotic analysis of displaced lunar orbits, J. of Guidance, Control, and Dynamics 32, 1666 (2009).

[75] J. Simo and C. R. McInnes, Displaced solar sail orbits: dynamics and applications, in 20th AAS/AIAA Space Flight Mechanics Meeting, San Diego, California, 2010.

[76] J. Simo and C. R. McInnes, Designing displaced lunar orbits using low-thrust propulsion, J. of Guidance, Control, and 
Dynamics 33, 259 (2010).

[77] J. Simo and C. R. McInnes, Feedback stabilization of displaced periodic orbits: application to binary asteroids, Acta Astronautica 96, 106 (2014).

[78] P. L. Bender et al., The lunar experiment, Science 182, 229 (1973).

[79] I. I. Shapiro, R. D. Reasenberg, J. F. Chandler, and R. W. Babcock, Measurement of the de Sitter precession of the moon: A relativistic three-body effect, Phys. Rev. Lett. 61, 2643 (1988).

[80] M. R. Pearlman, J. J. Degnan, and J. M. Bosworth, The international laser ranging service, Adv. Space Res. 30, 135 (2002).

[81] J. G. Williams, S. G. Turyshev, and D. H. Boggs, Progress in lunar laser ranging tests of relativistic gravity, Phys. Rev. Lett. 93, 261101 (2004).

[82] Z. Altamimi, X. Collilieux, J. Legrand et al., ITRF2005: A new release of the international terrestrial reference frame based on time series of station positions and earth orientation parameters, J. Geophys. Res. 112, B09401 (2007).

[83] R. March, G. Bellettini, R. Tauraso, and S. Dell'Agnello, Constraining spacetime torsion with the Moon and Mercury, Phys. Rev. D 83, 104008 (2011).

[84] R. March, G. Bellettini, R. Tauraso, and S. Dell'Agnello, Constraining spacetime torsion with LAGEOS, Gen. Relativ. Gravit. 43, 3099 (2011).

[85] S. Dell'Agnello et al., Creation of the new industry-standard space test of laser retroreflectors for the GNSS and LAGEOS, J. Adv. Space Res. 47, 822 (2011).

[86] S. Dell'Agnello et al., Fundamental physics and absolute positioning metrology with the MAGIA lunar orbiter, Exp. Astron. 32, 19 (2011).

[87] S. Dell'Agnello et al., Probing general relativity and new physics with lunar laser ranging, Nucl. Instr. Methods Phys. Res. A 692, 275 (2012).

[88] M. Martini, S. Dell'Agnello et al., MoonLIGHT: A USA-Italy lunar laser ranging retroreflector array for the 21st century, Planet. Space Sci. 74, 276 (2012).

[89] D. Currie, S. Dell'Agnello, G. O. Delle Monache, B. Behr, and J. G. Williams, A lunar laser ranging retroreflector array for the 21st century, Nucl. Phys. B Proc. Suppl. 243, 218 (2013).

[90] S. Dell'Agnello et al., Next-generation laser retroreflectors for GNSS, solar system exploration, geodesy, gravitational physics and earth-observation, in ESA Proc. Int. Conf. on Space Optics (Tenerife, Spain, Oct. 2014).

[91] D. Vokrouhlicky, A note on the solar radiation perturbations of lunar motion, Icarus 126, 293 (1997).

[92] T. Levi Civita, The Absolute Differential Calculus (Blackie \& Son, London, 1926; Dover, New York, 1977).

[93] C. W. Misner, K. S. Thorne, and J. A. Wheeler, Gravitation, (W. H. Freeman and Company, San Francisco, 1973)

[94] E. Battista, Extreme regimes in quantum gravity, PhD thesis (Naples University, 2016); arXiv:1606.04259.

[95] Y. Fourès-Bruhat, Théorème d'existence pour certains systèmes d'équations aux dérivées partielles non linéaires, Acta Mathematica 88, 141 (1952); English translation in the Max Planck Institute for History of Science preprint series, document 480 (2016).

[96] H. Poincaré, Collected Works, Vol. 1 (Gauthier-Villars, Paris, 1928; Jacques Gabais, Paris, 2011).

[97] G. Valiron, Functional Equations. Applications (Masson, Paris, 1945). 